\title{
AN UPDATED CHECKLIST OF THE ORCHIDS OF MAHARASHTRA, INDIA
}

\author{
JeEWAN Singh JaLAL \& J. JAYANTHI \\ Botanical Survey of India, Western Regional Centre, Pune - 411 001, Maharashtra, India \\ *Corresponding author's email: jeewan.orchid@gmail.com
}

\begin{abstract}
A checklist of the Orchidaceae of Maharashtra is presented based on herbarium collections and recent botanical explorations. This list comprises 32 genera with 106 taxa. The best represented genus is Habenaria with 23 species, followed by Dendrobium with eleven, Eulophia and Oberonia with seven and Peristylus with six. In Maharashtra, the total endemic orchid species are 41 spread over in 13 genera. Of these 30 species, six species are endemic to Peninsular India and five species are endemic to India. Generally orchids are found in the nine major habitats in Maharashtra of which the best representation was observed in the semi-evergreen forests (55 species) followed by moist deciduous forests (48 species), high altitude rocky plateaus ( 26 species) and dry deciduous forests $(25$ species). Flowering, habit, habitat, endemic status and distribution in Maharashtra are presented in the checklist.
\end{abstract}

KeY words: India, Maharashtra, orchids, Western Ghats

Introduction. The Maharashtra state of the Western India is known for its flowering plant wealth was explored since 18th century by Law, Graham, Nimmo, Sykes, Jacquemont, Gibson and Dalzell, followed by Birdwood, Nairne, Hallberg, Acland, Cooke, Woodrow, Gammie \& Ryan, etc. Graham was the pioneer to publish a Catalogue of Bombay Plants where he mentioned 31 species of orchids under 11 genera (Graham 1839). The following publication was by Dalzell and Gibson (1861) titled 'Flora of Bombay' that described 57 species of orchids under 23 genera. However, prior to this Dalzell separately published his floristic work in the form of articles in 'Hooker's Journal of Botany and Kew Garden Miscellany' from 1850 to 1852 . Nairne (1894) in his book 'Flowering Plants of Western India' mentioned 45 orchid species under 14 genera. Birdwood (1886) mentioned six species of orchids in his catalogue of the flora of Matheran. After that, he produced a catalogue of the flora of Mahabaleshwar and Matheran (Birdwood $1887)$ and mentioned 19 orchid species. Woodrow (1898-1900) documented 68 orchids in Flora of Western India. Gammie (1904a, b, 1906, 1907a, b, 1908a, b, 1909a, b, 1910, 1911a, b, 1912) published a series of papers in Journal of Bombay Natural History Society on orchids of the Bombay Presidency and included 61 species under 30 genera. Cooke
(1907) described 31 genera and 75 species of orchids from Bombay Presidency. Subsequently, Blatter and McCann (1931a, b, c, 1932) revised the orchid flora of Bombay Presidency and documented 132 species under 37 genera including many endemic orchids. They explored the dense forest of North Kanara district as well as Konkan area of Maharashtra and made collections. However, many of those species collected do not precisely indicate the localities and a majority of these collections are hardly available in Indian herbaria such as BSI, BLAT, CAL, etc.

Santapau (1903-1970) had a special interest on the flora of Western Ghats and his explorations had resulted in publication of several floras significant to this region. He critically re-investigated the Orchidaceae of Bombay State and published series of articles in Journal of Bombay Natural History Society in 1959-1963. Later on in 1966, these published articles were reprinted in the form of book "The Orchids of Bombay". The work enumerates a total of 118 taxa under 36 genera. The Botanical Survey of India (BSI) after its reorganization in 1956 established its Western Circle at Pune. Since then several BSI scientists have led plant exploration tours to different places of Maharashtra. Botanists from Agharkar Institute, Pune University, Shivaji University, Kolhapur and Dr. Babasaheb Ambedkar Marathwada 
University, Aurangabad have also made frequent plant collection in the state and published several new records and rediscoveries. In the last half century, a number of floras have been published on different regions and districts of Maharashtra. The Botanical Survey of India published a Flora of Maharashtra in 1996 and accounted for a total 113 orchid taxa under 34 genera. Later, M. R. Almeida also published a Flora of Maharashtra in 2009 and accounted for a total 157 taxa in 46 genera which included many cultivated species. Recently Pande et al. (2010) were able to report 99 species of orchids occurring in the wild in North Western Ghats (including Maharashtra, Goa and Karnataka) and lately, Barbhuiya and Salunkhe (2016) enumerated 122 taxa in 36 genera. Previous enumerations, Almeida (2009) and Barbhuiya and Salunkhe (2016) have included many taxa from cultivated source. Moreover, many species were included due to misidentifications, ignorance or lack of critical study. Thus, there is a need to provide a rationalized inventory on the orchids of Maharashtra.

\section{Materials and Methods}

Study Area.- The state of Maharashtra lies in the Western and Central part of India between the latitudes $22^{\circ} 1^{\prime}$ ' to $16^{\circ} 4^{\prime} \mathrm{N}$ and longitudes $72^{\circ} 6^{\prime}$ to $80^{\circ} 9^{\prime}$ ' E. It is bordered by Gujarat and the Union territory of Dadra and Nagar Haveli to the NorthWest, Madhya Pradesh to the North and NorthEast, Chhattisgarh to the East, Karnataka to the South, Telangana to the South East, and Goa to the SouthWest (Fig. 1). It occupies an area of 307,731 km2, which accounts for about $9.84 \%$ of the total area of India. The elevation ranges from sea level to $1646 \mathrm{~m}$ a.s.1. It comprises 36 districts and physiographically, this state may be divided into three natural divisions: the coastal strip (the Konkan), the Sahyadri or the Western Ghats and the Maharashtra Plateau. The 'Konkan' is a narrow strip of coastal land lying between the Arabian Sea and the Western Ghats. The western side of the Konkan region has a coastal length of about $720 \mathrm{~km}$, whereas the width of this region ranges from 30 to 60 $\mathrm{km}$. The area of the Western Ghats of Maharashtra is known as 'Sahyadris', with a total length of $750 \mathrm{~km}$ and average width of $80 \mathrm{~km}$. The western face of the Ghats is cut by deep ravines and canyons. Many of

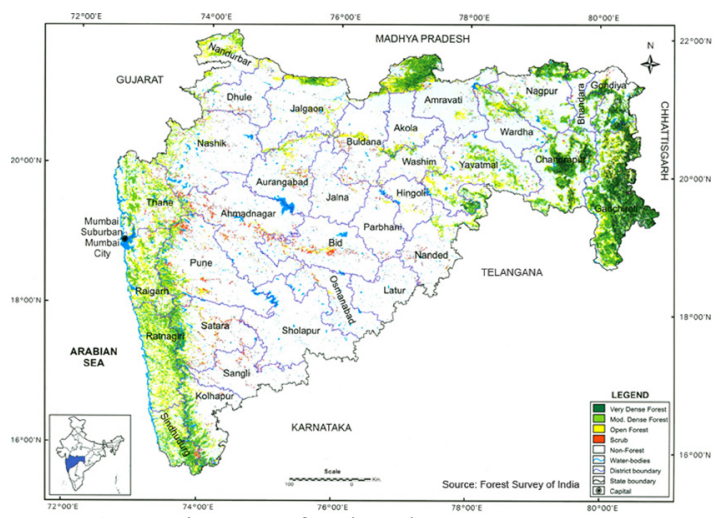

FIgURE 1. Location map of Maharashtra.

the peaks of the Western Ghats are more than 1400 $\mathrm{m}$ in height. Kalsubai $(1646 \mathrm{~m})$ is the highest peak in Maharashtra. Other important peaks are Salher (1567 m), Mahabaleshwar (1438 m), Saptashringi (1416 $\mathrm{m})$ and Trimbakeshwar $(1304 \mathrm{~m})$. The extensive tableland known as the Deccan or Maharashtra plateau is situated in the eastern region of Western Ghats. Over $90 \%$ of the area of the state is occupied by this plateau: the east-west stretch is $750 \mathrm{~km}$ long and the north-south width is about $700 \mathrm{~km}$, the average height is $450 \mathrm{~m}$. It is made of an impervious base of basaltic rocks overlaid with Deccan lavas. Tapi, Godavari, Bhima and Krishna are the main rivers of the state. This state has a tropical monsoon climate. Over $90 \%$ of the rainfall is due to the south-western monsoon (June to September). There is heavy rainfall in the coastal region (about $2000 \mathrm{~mm}$ ), scanty rains in the rain shadow areas in the centralparts (about $500 \mathrm{~mm}$ ) and medium rains in the easternparts (about $1000 \mathrm{~mm}$ ) of the state. As per Champion and Seth (1968) the State has 16 forest types, which belong to six forest type groups i.e., Tropical Semi-Evergreen, Tropical Moist Deciduous, Littoral and Swamp, Tropical Dry Deciduous, Tropical Thorn and Subtropical Broadleaved Hill Forests.

Data Collection. - The present checklist is an outcome of four years field visits from 2012 to 2015, supported with critical herbarium (CAL, BSI, BLAT, SUK, BAMU, Sardar Patel University Herbarium) studies and vast literature consultation (Almeida 1990, 2009, Bachulkar 2010, Bachulkar \& Yadav 1993, Bachulkar et al. 2011, Barbhuiya \& Salunkhe 2016, Birdwood 1886, 1887, Blatter 1908, 1909, 
1930, Blatter \& McCann 1931a,b,c, 1932, CerejoShivkar \& Shinde 2015, Cooke 1907, Datar \& Ghate 2012, Gaikwad et al. 2012, Gammie 1904a,b, 1906, 1907a,b, 1908a,b, 1909a,b, 1910, 1911a,b, 1912, Jalal \& Jayanthi 2013, Jalal et al. 2013, Jalal et al. 2014, Kamble \& Pradhan 1988, Kothari \& Moorthy 1993, Kulkarni 1988, Lakshminarasimhan \& Sharma 1991, Lakshminarasimhan et al. 1996, Mudaliar 1991, Nayar \& Kochhar 1984, Punekar 2002a,b, Sanghamitra et al. 2012, Santapau \& Kapadia 1966, Sardesai \& Yadav 2004, Sardeshi et al. 2002, Shah \& Badrinath 1985, Ugemuge 1986, Yadav \& Sardesai 2002, Yadav \& Sardesai 2002). A total of fifteen field visits of 10-20 days duration were undertaken to collect orchids from selected districts. Priority was given to those districts from where maximum numbers of orchid species have been reported (Fig. 2). A total of 21 districts were surveyed covering all the seasons in the year. Maximum field tours were undertaken during pre-monsoon (April-May) and monsoon (June-September) periods since this is the time with most orchid species flowering. All the collected specimens were processed with the standard herbarium technique (Jain \& Rao 1977). Specimens collected in vegetative conditions were brought and cultivated in the Conservatory of Botanical Survey of India, Pune for observation of flowering. After the study and identification, the standard online world databases, namely, Govaerts et al. (2016), Tropicos (2016), IPNI (2016) were also consulted for recent updates on the nomenclature and their global distributional details. Herbarium specimens collected during field work were deposited in the herbarium of Botanical Survey of India, Western Regional Centre, Pune (BSI).

\section{Results and Discussion}

This study has resulted in the documentation of 32 genera and 106 species for Maharashtra State. Of which, 53 species are epiphytic and 53 species are terrestrial, including one mycoheterotrophic (Epipogeum roseum). During the present survey 66 species $(62 \%)$ were studied alive and were found in the field, 30 species $(28 \%)$ were studied in herbarium and the remaining $10(9 \%)$ were included from literature with no voucher specimens available in

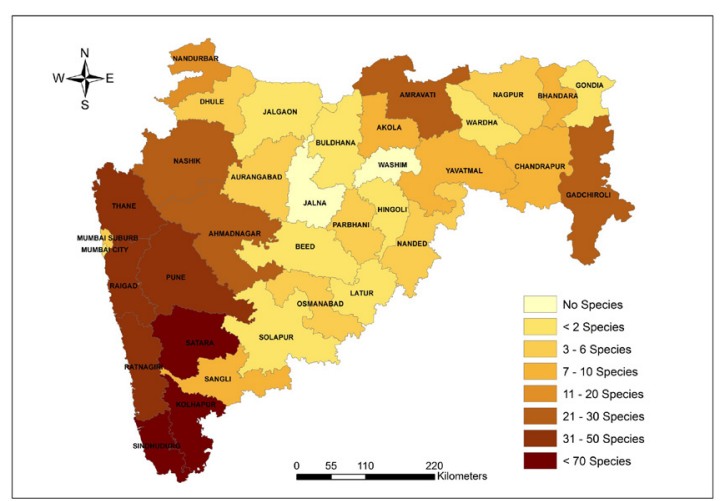

FIgURE 2. District wise representation of orchid richness in Maharashtra (data based on herbarium records and literature).

any Indian herbaria. Habenaria with 23 species is the largest genus in the state followed by Dendrobium (11), Eulophia and Oberonia (7) and Peristylus (6). In Maharashtra, the total endemic orchid species are 41 spread over in 13 genera. Of these, 30 species are endemic to Western Ghats (Bulbophyllum fimbriatum, B. sterile, B. stocksii, Conchidium filiforme, C. exile, C. microchilos, Dendrobium aqueum, D. barbatulum, D. lawianum, D. microbulbon, $D$. nanum, D. nodosum, D. ovatum, Gastrochilus flabelliformis, Habenaria elwesii, $H$. foliosa, $H$. heyneana, $H$. hollandiana, $H$. multicaudata, $H$. ovalifolia, H. rariflora, H. suaveolens, Oberonia brunoniana, O. verticillata, Pinalia mysorensis, $P$. polystachya, Plectoglossa perrottiana, Smithsonia maculata, S. straminea and S. viridiflora); six species are endemic to Peninsular India (Aerides maculosa, Eulophia pratensis, Habenaria brachyphylla, $H$. gibsonii, H. grandifloriformis and H. panigrahiana); and five species are endemic to India (Dendrobium herbaceum, Eria reticosa, Eulophia ochreata, Peristylus stocksii and Porpax jerdoniana). The concentrations of endemic orchids are observed mainly in high rainfall areas such as KhandalaLonavala, Mahabaleshwar-Koyna-Chandoli, Amboli and Radhanagari of Western Ghats. However, the Deccan plateau have a significantly lower number of species due to the little rainfall. The majority of the endemic species are confined to selected hill tops or small hill areas of semi-evergreen forests, plateaus and moist deciduous forests, making those areas very important for conservation. 

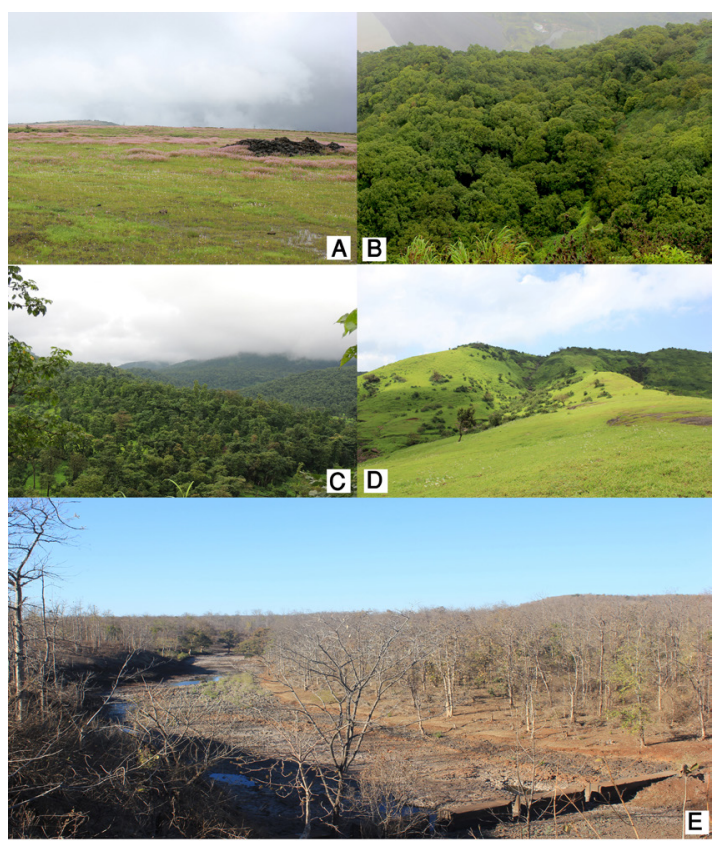

Figure 3. A. High altitude rocky plateau at Khambadev, Sindhudurg district, B. A view of semi-evergreen forest at Mahabaleshwar, C. Moist deciduous forest during rainy season at Kasara ghat, D. Mountain grassy slopes, E. Dry deciduous forest at Melghat Tiger Reserve, Amravati district

Habitats. Broadly orchids occur in the following major habitats in Maharashtra viz., semi-evergreen forests, moist deciduous forests, dry deciduous forest, coastal forests, high altitude rocky plateaus, low altitude rocky plateaus, mountain grassy slopes, semiarid grasslands and roadside avenue plantation (Fig. 3 ). Of the different habitats the semi-evergreen and moist deciduous forests harbors maximum number of species followed by plateaus and dry deciduous forests (Table 1).
Semi-evergreen forests. These forests are mostly occurring in patches along the upper elevations between $500-1000 \mathrm{~m}$. This is an important vegetation type having rainfall about $2000-4000 \mathrm{~mm}$ and a dry season of seven months. This forest type includes secondary evergreen dipterocarp forests, lateritic semi-evergreen forests, bamboo brakes, and riparian forests as described by Champion and Seth (1968). Important trees observed in semi-evergreen forests are Terminalia chebula, T. bellirica, Alstonia scholaris, Careya arborea, Holigarna arnottiana, H. grahamii, Macaranga peltata, Mangifera indica, Mitragyna parviflora, Syzygium cumini, Ixora brachiata and Kydia calycina. Epiphytic orchids such as Aerides crispa are found growing on medium sized trees in open canopies. Similarly, endemic orchid Smithsonia viridiflora which grows on tree trunks and is found in the open canopy area. Interesting examples like Chierostylis parvifolia, which is a ground orchid but sometimes is found growing as epiphytes on the crevices of moss laden trunks. Dendrobium aqueum and D. microbulbon are also found growing as epiphytes on branches of small trees. Between the gaps of semi-evergreen forest patches, Habenaria brachyphylla has found suitable habitat for growth. Peristylus stocksii, P. plantagineus, $P$. aristatus, Habenaria ovalifolia and Malaxis versicolor are generally found in undergrowth of evergreen forests. Zeuxine affinis, Z. longilabris and Habenaria digitata grow under the shade of semi-evergreen forests. Strobilanthes callosus is one of the dominant shrubs on the outskirts of semi-evergreen forests. Habenaria heyneana and Pecteilis gigantea are found along the margins of Strobilanthes callosus patches.

TABLE 1. Orchid species richness in different habitats.

\begin{tabular}{|c|c|c}
\hline SI. No. & Habitat types & Species richness \\
\hline 1 & Semi-evergreen forests & 45 \\
\hline 2 & Moist deciduous forests & 25 \\
\hline 3 & Dry deciduous forests & 3 \\
\hline 4 & Coastal forests & 26 \\
\hline 5 & High altitude rocky plateaus & 10 \\
\hline 6 & Low altitude rocky plateaus & 9 \\
\hline 7 & Mountain grassy slopes & 2 \\
\hline 8 & Semi-arid grassland & 6 \\
\hline
\end{tabular}


Moist deciduous forests. Moist deciduous forests are one of the common vegetation types at the base of Western Ghats and Konkan region with high rainfall of $1500-2500 \mathrm{~mm}$. The rainfall is restricted to south-west monsoon. The common trees are Albizia chinensis, Canthium dicoccum, Careya arborea, Catunaregam spinosa, Dalbergia latifolia, Diospyros chloroxylon, Dillenia pentagyna, Ficus racemosa, Macaranga peltata, Madhuca longifolia, Mallotus philippensis, Meyna laxiflora, Syzygium cumini, Terminalia paniculata and Xylia xylocarpa, etc. The percentage of epiphytic species is more prevalent in moist deciduous forests than compared to other forests types. The top canopy trees shed their leaves in winter, then the epiphytic species start flowering soon after this phenomenon. Epiphytic orchids such as Aerides maculosa, Conchidium microchilos, Dendrobium barbatulum, D. ovatum, D. peguanum, Oberonia recurva and common ground orchids such as Eulophia spectabilis, Geodorum densiflorum, Habenaria digitata, $H$. foliosa, $H$. gibsonii, Nervilia concolor, N. crociformis, Pecteilis gigantea, Peristylus lawii and P. plantagineus are commonly found in this habitat.

Dry deciduous forests. This type of forests comprises mixed tree species which remain deciduous for several months during the dry season. Teak predominates in this type of habitat. The rainfall varies from $90-1300$ $\mathrm{mm}$. Trees such as Tectona grandis, Anogeissus latifolia, Bombax ceiba, Boswellia serrata, Holoptelea integrifolia, Lagerstroemia parviflora, Lannea coromandelica, Hardwickia binata, Butea monosperma, Erythrina stricta, Sterculia urens etc. are common hosts for many epiphytic orchids such as Rhynchostylis retusa, Vanda tessellata and V. testacea. In the rainy season ground orchids such as Eulophia spectabilis, Habenaria furcifera, $H$. plantaginea, $H$. roxburghii, Nervilia concolor, $N$. crociformis, and Pecteilis gigantea are commonly found.

Coastal forests. Although in Maharashtra there is no well defined coastal forest, some regions along the west coast still harbor mangrove vegetation. They are mainly found along the estuaries along the coasts of Greater Bombay, Raigad, Ratnagiri, Sindhudurg and Thane districts. All along the coastline there are tidal swamps and mudflats mostly covered by mangroves. Species such as Avicennia officinalis, Ceriops tagal, Rhizophora mucronata, Sonneratia apetala, Lumnitzera racemosa, Excoecaria agallocha, Xylocarpus granatum, Barringtonia racemosa and Thespesia populnea are common. In some localities along Konkan the vegetation is also mixed with semi-evergreen elements. Orchids such as Acampe praemorsa, Aerides maculosa and Cottonia peduncularis are infrequently seen in this type of vegetation.

High altitude rocky plateaus. These habitats are the characteristic features of Western Ghats of Maharashtra. These laterites are most typically hardened flat topped hills, also known as tablelands. These have an elevation range between 800 and 1400 $\mathrm{m}$, and are found in the districts of Pune, Satara, Kolhapur, Sangli, Ratnagiri and Sindhudurg. Lateritic rock shows brown and black colors because of the high concentration of ferrous and aluminum. The soil formation on the outcrops is extremely slow and the soil depth varies from a few centimeters on flat areas to about a meter in deep cracks and depressions. The soil is sandy to sandy loam in texture, highly acidic and poor in phosphates. Fourteen orchid species were reported in these high altitude rocky plateaus, of these seven species are epiphytic and the rest are ground orchids. In many plateaus where the soil depth is about $1 \mathrm{~m}$, there is support for stunted host trees such as Memecylon umbellatum which forms forest patches. These stunted forest patches support many epiphytic orchids such as Aerides crispa, A. maculosa, Bulbophyllum fimbriatum, Conchidium filiforme, Dendrobium aqueum, D. barbatulum, D. herbaceum, D. microbulbon and Oberonia recurva. Sometimes epiphytic species are also seen growing on the boulders as lithophytes. Boulders are found on the plateau, either aggregated or scattered. Conchidium reticosum always grows on small boulders on plateaus. The shady surface also supports terrestrial orchids such as Habenaria brachyphylla, H. foliosa, H. gibsonii, Liparis odorata, Malaxis versicolor, Nervilia crociformis, $N$. infundibulifolia, Pecteilis gigantea, Peristylus aristatus, $P$. plantagineus and $P$. stocksii. The moss and duff covering these boulders allows sometimes for terrestrial orchids to grow 
too. During the monsoon these plateaus provides many microhabitats for endemic ground orchids. In Mahabaleshwar, Panchgani and Chandgad area one can see the mass flowering of thousands of Habenaria suaveolens, $H$. grandifloriformis, $H$. heyneana, $H$. rariflora and Peristylus densus.

Low altitude rocky plateaus. This particular habitat occurs along the Konkan region between 20-200 m in Raigad, Ratnagiri and Sindhudurg districts. The coastal laterites of Konkan region are derived from the materials deposited from erosion of the Western Ghats and in-situ formation from low level basalts, mixed together. It covers the largest land surface in the central and southern Konkan, and these are locally known as sadas (Watve 2013). It is also a recognised habitat category under the IUCN habitat classification. The rocky plateaus in the Konkan region and the southern parts of the Northern Western Ghats have basalt as their base rock, capped with laterite. The laterites are normally subjected to alternate wet and dry climates, appearing totally barren during the dry season and followed by drastic changes in physiognomy over a period of four months during the rainy season (Prabhu 2013). The early phase of the monsoon is the period of vegetative growth of many plants including orchids. Ten orchid species including three endemic orchids are reported in this habitat. The gregarious populations of Habenaria grandifloriformis are seen during the month of June. In mid August, Habenaria diphylla and H. marginata are seen. The tubers of these ground orchids remain almost eight months in a dormant stage during the dry season. Tubers are modified organs that have nutrient storage for growth of new plants in the next growing season. Due to the thin layer of soil in plateaus, the tubers size reach only about $0.5-1 \mathrm{~cm}$.

Semi-arid grassland. Two types of grassland habitats are reported in the study area. One is mountain grassy slopes which are found along the mountain slopes. These mountain grassy slopes though dominated by grasses are also rich in orchid diversity. Orchids such as Habenaria commelinifolia, $H$. diphylla, $H$. grandifloriformis, $H$. heyneana, $H$. longicorniculata and Pecteilis gigantea are recorded from these grasslands. Another grassland habitat is found in the
Deccan plateau region. The grasslands of the Deccan plateau are of the semi-arid type with dry climatic conditions. These grasslands occur in districts such as Ahmednagar, Solapur, and Osmanabad, etc. Orchid diversity in this grassland is very poor, represented by a few species such as Eulophia graminea, E. ochreata, E. pratensis and Zeuxine strateumatica.

Roadside avenue plantation. Other than different forest habitats, orchids are also found in plantations, orchards, and roadside trees in different parts of Maharashtra which receives considerable rainfall. Large colonies of Acampe praemorsa were found growing on roadside trees such as Samanea saman, Tamarindus indica, and Ficus benghalensis. Other epiphytic orchids such as Aerides maculosa, Dendrobium barbatulum, D. ovatum, Cottonia peduncularis can be found growing in mango plantations, and cashew plantations. In Panchgani, Chandgad and Bhimashankar where rainfall is high some epiphytic orchids such as Dendrobium barbatulum, Aerides maculosa and Conchidium filiforme are found growing in Eucalyptus globulus plantations.

\section{Host species diversity of epiphytic orchids.} The present study reports a total 58 host plants of 29 species of epiphytic orchids. Dendrobium barbatulum is one endemic orchid that shows diverse host ranges and can be found growing on more than 29 host trees, followed by Aerides maculosa and $D$. ovatum which can be found to grow adding on 25 and 22 host trees respectively. They show their tolerance to wideranging microclimatic conditions available over the host trees. Epiphytic orchids, that are found on less than 10 host tree species constituted 58\% of the total epiphytic species. Out of 58 host tree species recorded with epiphytic orchids, Mangifera indica supports 20 species of orchids. Other host tree species more important for epiphytic orchids are Careya arborea, Terminalia bellirica, T. chebula, and Syzygium cumini, and they supported about 13 to 15 species respectively. In the plateaus the most suitable host is Memecylon umbellatum. In many areas Catunaregam spinosa was found to be a suitable host for Dendrobium barbatulum. In the dry deciduous forests of Satpura range epiphytic orchids such as Rhynchostylis retusa, Vanda tessellata and V. testacea 
are commonly seen on Diospyros melanoxylon, Holoptelea integrifolia, Lagerstroemia parviflora, Madhuca longifolia var. latifolia, Ougeinia oojeinensis, Stereospermum chelonoides, Tectona grandis and Ziziphus mauritiana. It was also observed that in the Konkan region Acampe praemorsa can be found growing on the main trunk of Cocos nucifera. Similarly, two other interesting host species were also reported such as Phoenix sylvestris and Euphorbia neriifolia. In high rainfall pockets Eucalyptus citriodora and Eucalyptus globulus also support some epiphytic orchids.

Threats. Habitat degradation is the main threat to the orchids in Maharashtra. Orchids populations are also threatened by habitat loss and unsustainable exploitation. A decline in the number of orchid species is reported from Panchgani, Kas Plateau and Khandala. Kas plateau, known as the valley of flowers, is facing a surge of tourists. Excited visitors take the orchids for their homes, leaving little chance for these rare orchids to survive. The fragrant Pecteilis gigantea popularly known as the queen of Khandala was found very commonly fifty years ago and sold in the Khandala hill station's markets. This led to a fall in the species population and now it is confined to a few spots only. Likewise, Habenaria suaveolens (with popular synonym Habenaria panchganiensis) popularly known as Panchgani orchid, was once abundant in Panchgani plateau and now became a rare sight due to the tourism activities such as horse rides and camel rides that almost converted the flora rich plateau to a barren land. In Konkan region many of the good forests patches have been cleared for cash crops such as Areca nut, Cashew nut and mango orchards. Likewise, grazing also damages several terrestrial orchids in plateau areas. Eulophia spectabilis is a terrestrial orchid which is being extracted from wild leading to drastic depletion of wild populations. It is commonly known as Amarkanda and is widely used to cure various health problems and ailments. The corm of the plant is used in the preparation of 'salep', which is taken as an aphrodisiac (Jalal et al. 2014). In many locations in Maharashtra, orchids are also facing threats due to the invasion of alien weeds such as Eupatorium odoratum, Mikania cordata, Lantana camara and Parthenium hysterophorus.

In the present study the entire list of species is arranged alphabetically. For each taxon, the current name with its basionym is provided, followed by a full reference to the original publication and further synonyms (restricted to those that were used in the relevant literature). Flowering months of the taxon, life form, habitat, distribution in Maharashtra, global distribution and specimen examined are given. At the end, excluded taxa are given with justification.

\section{H E C K L I S T}

\section{ACAmpe Lindl.}

Acampe praemorsa (Roxb.) Blatt. \& McCann, J. Bombay Nat. Hist. Soc. 35: 495. 1932. Fig. 4A. Epidendrum praemorsum Roxb., Pl. Coromandel 1: 34, t. 43. 1795.

Flowering: April-June.

LIFE FORM: Epiphyte.

HaвiтAT: Found in the moist deciduous forests, dry deciduous forests and coastal forests.

Distribution IN Maharashtra: Gadchiroli, Kolhapur, Mumbai Suburban, Raigad, Ratnagiri, Satara, Sindhudurg and Thane.

General Distribution: India (Andhra Pradesh, Odisha, Goa, Gujarat, Maharashtra, Karnataka, Kerala, Tamil Nadu Daman \& Diu, Dadara \& Nagar Haveli, Jharkhand, Chhattisgarh,
Madhya Pradesh, Rajasthan), Nepal, Sri Lanka, Myanmar.

SPECIMENS EXAmined: Gadchiroli district, Surajgad, R.S.Govekar 1652 (BAMU). Kolhapur district, Udegiri, M.M.Sardesai 1723 (SUK). Mumbai Suburban district, Borivali, P.S.Herbert 1615 (BLAT). Raigad district, Alibagh, J.S.Jalal 200875 (BSI). Ratnagiri district, Mangaon, J.S.Jalal 194905 (BSI). Satara district, Koyna, J.S.Jalal 200792 (BSI). Sindhudurg district, Sawantwadi, J.S.Jalal 194993 (BSI). Thane district, Mal, J.S.Jalal 200729 (BSI).

Acampe ochracea (Lindl.) Hochr., Bull. New York Bot. Gard. 6: 270. 1910.

Saccolabium ochraceum Lindl., Edwards's Bot. Reg. 28(Misc.): 2 (1842). 
Flowering: May - August.

LIFE FORM: Epiphyte.

Distribution in Maharashtra: Raigad.

General distribution: India (Assam, Manipur, Meghalaya, Mizoram, Nagaland, Arunachal Pradesh, Sikkim, West Bengal, Andhra Pradesh, Odisha, Karnataka, Kerala, Tamil Nadu), Bangladesh, Bhutan, Nepal, Sri Lanka, China, Myanmar, Thailand, Indo-China.

Note: This species is included based on Kothari and Moorthy (1993) who reported it from Karnala fort in Raigad district. There is no herbarium specimen traced in herbarium BSI to confirm its occurrence in Maharashtra.

\section{Aerides Lour.}

Aerides crispa Lindl., Gen. Sp. Orchid. Pl.: 239. 1833. Fig. 4B.

FLOWERING: April-June.

LIFE FORM: Epiphyte.

HaвiтAт: Found in semi-evergreen forests.

Distribution in Maharashtra: Kolhapur, Pune, Ratnagiri, Satara, Sindhudurg and Thane.

General Distribution: India (Goa, Gujarat, Karnataka, Kerala, Maharashtra, Tamil Nadu, Dadara \& Nagar Haveli), Bangladesh.

Specimens EXAmined: Kolhapur district, Radhanagari, J.S.Jalal 200518 (BSI). Pune district, Lonavala, J.S.Jalal 200406 (BSI). Ratnagiri district, Kudavale, J.S.Jalal 194917 (BSI). Satara district, Mahabaleshwar, J.S.Jalal 200806 (BSI). Sindhudurg district, Amboli, J.S.Jalal 200492 (BSI). Thane district, Tokavada range, K.V.Billore 115624 (BSI).

Aerides maculosa Lindl., Edwards's Bot. Reg. 31: 58.

1845. Fig. 4C.

FLOWERING: May - July.

LIFE FORM: Epiphyte.

Habitat: Found growing in moist deciduous, drydeciduous and semi-evergreen forests.

Distribution IN MAHARAshtra: Amravati, Gadchiroli, Kolhapur, Nandurbar, Nashik, Pune, Raigad, Ratnagiri, Satara, Sindhudurg and Thane.

General Distribution: India (Andhra Pradesh, Odisha, Goa, Gujarat, Maharashtra, Karnataka, Kerala, Tamil Nadu, Madhya Pradesh,
Rajasthan), endemic to Peninsular India.

SPECIMENSEXAMINED:Amravatidistrict, Chikhaldara, J.S.Jalal 200736 (BSI). Gadchiroli district, Jharapapada, R.S.Govekar 1671 (BAMU). Kolhapur district, Radhanagari, J.S.Jalal 200532 (BSI). Nandurbar district, Toranmal, J.S.Jalal 195112 (BSI). Nashik district, Kasara Ghat, J.S.Jalal 200874 (BSI). Pune district, Lonavala, J.S.Jalal 200406 (BSI). Raigad district, Phansad WLS, J.S.Jalal 200883 (BSI). Ratnagiri district, Dapoli, J.S.Jalal 194926 (BSI). Satara district, Mahabaleshwar, J.S.Jalal 200812 (BSI). Sindhudurg district, Amboli, J.S.Jalal 194954 (BSI). Thane district, Tansa, J.S.Jalal 200853 (BSI).

Aerides multiflora Roxb., Pl. Coromandel 3: 68. 1820. FLOWERING: May - October.

LIFE FORM: Epiphyte.

HaBitat: Found growing in dry-deciduous forest.

Distribution In Maharashtra: Gadchiroli.

General distribution: India (Assam, Manipur, Meghalaya, Mizoram, Nagaland, Tripura, Arunachal Pradesh, Sikkim, West Bengal, Uttarakhand, Himachal Pradesh, Odisha, AndhraPradesh,Madhya Pradesh, Chhattisgarh, Rajasthan, Andaman \& Nicobar), Bangladesh, Bhutan, Nepal, Myanmar, Thailand, IndoChina.

Specimen eXAmined: Gadchiroli district, Gatta, R.S.Govekar s.n. (BAMU).

Aerides odorata Lour., Fl. Cochinch.: 525. 1790.

FLowerING: May-June.

LIFE FORM: Epiphyte.

HАBITAT: Found growing in moist deciduous forest. Distribution in Maharashtra: Gadchiroli.

General distribution: India (Assam, Manipur, Meghalaya, Mizoram, Nagaland, Tripura, Arunachal Pradesh, Sikkim, West Bengal, Uttarakhand, Odisha, Andhra Pradesh, Karnataka, Madhya Pradesh, Chhattisgarh, Andaman \& Nicobar), Bangladesh, Bhutan, Nepal, Myanmar, Malaysia, Indonesia, Philippines, Thailand, Indo-China.

Specimen eXAmined: Gadchiroli district, Kawande, R.S.Govekar 1692 (BAMU).

Aerides ringens (Lindl.) C.E.C.Fisch. in J.S.Gamble, Fl. Madras: 1442. 1928. 
Saccolabium ringens Lindl., Gen. Sp. Orchid. Pl.: 220. 1833.

Flowering: May-July.

LIFE FORM: Epiphyte.

Distribution In Maharashtra: Thane and Satara.

General Distribution: India (Andhra Pradesh, Odisha, Goa, Gujarat, Karnataka, Kerala, Tamil Nadu), Sri Lanka.

Note: This species is included here based on Dalzell and Gibson (1861) collection from Salsette and Woodrow's collection from Mahabaleshwar. Mulgaonkar and Dabhade (2005) reported it from Panvel in Raigad District. However, there is no specimen available in any of the herbaria. Recently it was reported from Valsad district of Gujarat near Maharashtra border by Bhatt and Nagar (2014). There is a possibility of its occurrence in Maharashtra.

\section{Bulbophyllum Thouars}

Bulbophyllum fimbriatum (Lindl.) Rchb.f. in W.G.Walpers, Ann. Bot. Syst. 6: 260. 1861. Fig. 4D. Cirrhopetalum fimbriatum Lindl., Edwards's Bot. Reg. 25(Misc.): 72.1839.

FLOWERING: December-May.

LIFE FORM: Epiphyte.

HABITAT: Found in semi-evergreen forests.

Distribution in Maharashtra: Pune, Satara and Sindhudurg.

General Distribution: India (Goa, Karnataka, Kerala, Maharashtra, Tamil Nadu), endemic to Western Ghats.

SPeCimENS EXAmined: Pune district, Junnar, J.S.Jalal 200831 (BSI). Sindhudurg district, Bhedshi, B.G.Kulkarni 120453 (BSI). Satara district, Mahabaleshwar, J.S.Jalal 200810 (BSI).

Bulbophyllum sterile (Lam.) Suresh in D.H.Nicolson, C.R.Suresh \& K.S.Manilal, Interpret. Van Rheede's Hort. Malab.: 298. 1988. Fig. 4E.

Epidendrum sterile Lam., Encycl. 1: 189. 1783.

Bulbophyllum nilgherrense Wight, Icon. P1. Ind. Orient. 5: t. 1680. 1851.

LIFE FORM: Epiphyte.

Flowering: December-May.

HАвітAт: Found in semi-evergreen forests.

Distribution in Maharashtra: Sindhudurg and Kolhapur.
General distribution: India (Andhra Pradesh, Goa, Karnataka, Kerala, Maharashtra, Tamil $\mathrm{Nadu}$, endemic to Western Ghats.

Specimens examined: Kolhapur district, Patgaon, M.M.Sardesai 2027 (SUK). Sindhudurg district, Konshi, Sawantwadi, S.M.Almeida 5232 (BLAT).

Bulbophyllum stocksii (Benth. ex Hook.f.) J.J.Verm., Schuit. \& de Vogel, Phytotaxa 166: 111. 2014.

Trias stocksii Benth. ex Hook.f., Fl. Brit. India 5: 781. 1890.

FLOWERING: February-March.

LIFE FORM: Epiphyte.

Distribution In Maharashtra: Konkan at c. 100 m. General Distribution: India (Maharashtra, Karnataka, Kerala and Tamil Nadu), endemic to Western Ghats.

SPeCimens eXAmined: Konkan “Concan”, Stocks \& Law s.n. (GH-00287630, GOET-008707).

Note: It is included on the authority of J.D. Hooker (1980). He mentioned it based on the collection by Law from south and north Konkan region. It has not been recollected by any subsequent workers from Maharashtra region. There is no herbarium record of its collection from Maharashtra.

\section{Cheirostylis Blume}

Cheirostylis flabellata (A.Rich.) Wight, Icon. P1. Ind. Orient. 5: 16. t. 1727. 1851.

Goodyera flabellata A.Rich., Ann. Sci. Nat., Bot., ser. 2, 15: 79. 1841.

Monochilus flabellatus (A.Rich.) Wight, Icon. P1. Ind. Orient. 5: t. 1727. 1851.

FLOWERING: November.

LIFE FORM: Terrestrial.

HАвітAт: Found under the shade of semi-evergreen forests.

Distribution in Maharashtra: Satara.

General Distribution: India (Karnataka, Kerala, Maharashtra, Tamil Nadu), Sri Lanka, Myanmar.

SPeCimen eXAmined: Satara district, Mahabaleshwar, P.V.Bole 2244 (BLAT).

Cheirostylis parvifolia Lindl., Edwards's Bot. Reg. 25(Misc.): 19. 1839.

FLOWERING: November-February.

LIFE FORM: Terrestrial. 
HABITAT: Found under the shade of semi-evergreen forests.

Distribution in Maharashtra: Kolhapur, Satara and Sindhudurg.

General Distribution: India (Kerala, Maharashtra, Odisha, Tamil Nadu), Sri Lanka.

SPecimens eXamined: Kolhapur district, Chandgad, J.S.Jalal 197709 (BSI). Satara district, Koyna, Prajakta Pathare 198950 (BSI). Sindhudurg district, Sawantwadi, S.M.Almeida s.n. (BLAT!).

\section{Cleisostoma Blume}

Cleisostoma tenuifolium (L.) Garay, Bot. Mus. Leafl. 23: 175. 1972.

Epidendrum tenuifolium L., Sp. P1.: 952. 1753.

FLOWERING: June-November.

LIFE FORM: Epiphyte.

НАвітAT: Found in semi-evergreen forests.

Distribution in Maharashtra: Sindhudurg.

General Distribution: India (Goa, Karnataka,

Kerala, Maharashtra, Tamil Nadu), Sri Lanka, Thailand.

Specimen EXAmined: Sindhudurg district, Sawantwadi, S.M.Almeida 4948 (BLAT).

\section{Conchidium Griff.}

Conchidium filiforme (Wight) Rauschert, Feddes Repert. 94: 444. 1983. Fig. 5J.

Eria filiformis (Wight) Rchb.f. in W.G.Walpers, Ann. Bot. Syst. 6: 268. 1861.

Dendrobium filiforme Wight, Icon. Pl. Ind. Orient. 5: t. 1642.1851.

Eria dalzellii (Hook. ex Dalzell) Lindl., J. Proc. Linn. Soc., Bot. 3: 47.1858 (nom. et syn. tantum, non descr.).

Eria conrardii M.R.Almeida, Fl. Maharashtra 5A: 42. 2009.

Eria conrardii var. fimbriata (Hook.f.) M.R.Almeida, Fl. Maharashtra 5A: 43. 2009.

FLowering: July-August.

LIFE FORM: Epiphyte.

Haвitat: Found in moist-deciduous and semievergreen forests.

Distribution in Maharashtra: Kolhapur, Pune, Raigad, Ratnagiri, Sindhudurg, Satara and Thane.
General Distribution: India (Gujarat, Goa, Karnataka, Kerala, Maharashtra, Tamil Nadu), endemic to Western Ghats.

SPeCimens EXAmined: Kolhapur district, Radhanagari, J.S.Jalal 200531 (BSI). Pune district, Lonavala, J.S.Jalal 200409 (BSI). Raigad district, Matheran, J.S.Jalal 200585 (BSI). Ratnagiri district, Dapoli, J.S.Jalal 194940 (BSI). Satara district, Mahabaleshwar, J.S.Jalal 200411 (BSI). Sindhudurg district, Khamdadev plateau, J.S.Jalal 195013 (BSI). Thane district, Tansa, J.S.Jalal 200859 (BSI).

Conchidium exile (Hook.f.) Ormerod, Taiwania 57: 119. 2012. Fig. 5I.

Eria exilis Hook. f., Fl. Brit. India 5: 788. 1890.

LeAves: June-October; Flowering: OctoberDecember.

LIFE FORM: Epiphyte.

НАвітAт: Found in semi-evergreen forests on moss laden tree trunks.

Distribution in Maharashtra: Kolhapur, Pune, Satara, Sindhudurg.

General Distribution: India (Goa, Kamataka, Kerala, Maharashtra, Tamil Nadu), endemic to Western Ghats.

SPECimENS EXAmined: Kolhapur district, Chandgad, J.S.Jalal 195199 (BSI). Pune district, Khandala, Z.J.Kapadia 2297 (BLAT). Satara district, Mahabaleshwar, J.S.Jalal 200433 (BSI). Sindhudurg district, Khamdadev plateau, J.S.Jalal 195014 (BSI).

Conchidium microchilos (Dalzell) Rauschert, Feddes Repert. 94: 444. 1983. Fig. 5K.

Dendrobium microchilos Dalzell, Hooker's J.

Bot. Kew Gard. Misc. 3: 345. 1851. Eria microchilos (Dalzell) Lindl., J. Proc. Linn. Soc., Bot. 3: 47. 1858.

FLowering: July-August.

LIFE FORM: Epiphyte.

HaBitat: Found in moist-deciduous forests and semi-evergreen forests.

Distribution in Maharashtra: Kolhapur, Pune, Raigad, Satara and Sindhudurg.

General distribution: India (Goa, Karnataka, Kerala, Maharashtra, Tamil Nadu), endemic to Western Ghats.

SPECimens EXAmined: Kolhapur district, 
Radhanagari, J.S.Jalal 200516 (BSI). Pune district, Amby valley, J.S.Jalal 200570 (BSI). Raigad district, Matheran, J.S.Jalal 200589 (BSI). Satara district, Mahabaleshwar, P.V.Bole 329 (BLAT). Sindhudurg district, Amboli, J.S.Jalal 195022 (BSI).

Conchidium reticosum (Wight) Ormerod, Taiwania 57: 119. 2012. Fig. 6A.

Eria reticosa Wight, Icon. Pl. Ind. Orient. 5(1): 4, t. 1637.1851.

Conchidium braccatumauct. non. (Lindl.) Brieger: R. Govaerts, World Checkl. Monocot. 2014 (only syn. E. reticosa Wight \&E. uniflora Dalzell).

Flowering: May-June.

LIFE FORM: Epiphyte.

HABITAT: Found in semi-evergreen forests between $600-1200 \mathrm{~m}$ and rarely seen growing on trees but common occurrence on rocky surfaces in high altitude plateaus.

Distribution in Maharashtra: Kolhapur, Pune, Satara, Sindhudurg and Thane.

General Distribution: India (Uttarakhand, Goa, Karnataka, Kerala, Maharashtra, Tamil Nadu), endemic to India.

SPecimens EXAmined: Kolhapur district, Borbet, M.M.Sardesai 168 (SUK). Pune district, Ambe plateau, Junnar, J.S.Jalal 200823 (BSI). Satara district, Kas, M.P.Bachulkar 5437 (SUK). Sindhudurg district, Khamdadev plateau, J.S.Jalal 195012 (BSI).

\section{Cottonia Wight}

Cottonia peduncularis (Lindl.) Rchb.f., Cat. OrchidsSamml. Schiller, ed. 3: 22. 1857. Fig. 4F.

Vanda peduncularis Lindl., Gen. Sp. Orchid. Pl.: 216. 1833.

FLOWERING: March-April.

LIFE FORM: Epiphyte.

НАвітAт: Found in moist-deciduous forests and semi-evergreen forests.

Distribution in Maharashtra: Kolhapur, Nashik, Raigad, Ratnagiri, Sindhudurg and Thane.

General Distribution: India (Odisha, Goa, Karnataka, Kerala, Maharashtra Tamil Nadu), Sri Lanka.

Specimens eXamined: Nashik district, Igatpuri,
J.S.Jalal 195152 (BSI). Raigad district, Talerwarphatta, J.S.Jalal 194901 (BSI). Ratnagiri district, Vetadvadi, Dapoli, J.S.Jalal 194927 (BSI). Sindhudurg district, Sawantwadi, J.S.Jalal 195023 (BSI). Thane district, Usgaon, N.Y.Das 3198 (BLAT).

\section{Cymbidium Swartz}

Cymbidium aloifolium (L.) Sw., Nova. Acta. Regial Soc. Sci. Upsal. 6: 73. 1799. Epidendrum aloifolium L., Sp. Pl. 953. 1753.

FLowering: May-June.

LIFE FORM: Epiphyte.

Habitat: Found in the moist-deciduous forests at elevations between $50-100 \mathrm{~m}$.

Distribution in Maharashtra: Kolhapur and Sindhudurg.

General Distribution: India (Assam, Manipur, Meghalaya, Mizoram, Nagaland, Tripura, Arunachal Pradesh, Sikkim, West Bengal, Uttarakhand, Goa, Karnataka, Kerala, Maharashtra, Tamil Nadu, Bihar, Chhattisgarh, Jharkhand, Madhya Pradesh, Andaman \& Nicobar), Bangladesh, Nepal, Sri Lanka, China, Myanmar, Indonesia, Malaysia, Thailand, Indo-China.

SPecimens eXamined: Kolhapur: Patgaon, M.M.Sardesai 2031 (SUK). Sindhudurg: Amboli, P.D.Mahekar s.n. (SUK).

Cymbidium bicolor Lindl., Gen. Sp. Orchid. Pl.: 164. 1833. Fig. 4G.

FLOWERING: May-June.

LIFE FORM: Epiphyte.

HABIтAT: Found in the moist-deciduous forests at elevations between 50-100 m.

Distribution in Maharashtra: Sindhudurg.

General Distribution: India (Odisha, Goa, Karnataka, Kerala, Maharashtra, Tamil Nadu), Nepal, Sri Lanka.

Specimen examined: Sindhudurg district, Pulas, Sawantwadi J.S.Jalal 195027 (BSI).

\section{Dendrobium Swartz}

Dendrobium aqueum Lindl., Edwards's Bot. Reg. 28: Misc. 5. 1843.

Flowering: September-October. 
LIFE FORM: Epiphyte.

HaвiтAт: Found in semi-evergreen forests at elevations between 100-1300 m.

Distribution in Maharashtra: Ahmednagar, Kolhapur, Nashik, Pune, Satara and Sindhudurg.

General Distribution: India (Andhra Pradesh, Goa, Karnataka, Kerala, Maharashtra, Tamil $\mathrm{Nadu}$ ), endemic to Western Ghats

Specimens eXamined: Ahmednagar district, Pachnai plateau, Harishchandragad, K.V.Billore 115751 (BSI). Kolhapur district, Ajara, J.S.Jalal 200490 (BSI). Nashik district, Ambewadi, P.L. Narasimhan 167518 (BSI). Pune district, Durga khilla, Junnar, Hemadri 89935 (BSI). Sindhudurg district, Amboli, J.S.Jalal 194962 (BSI). Satara district, Mahabaleshwar, J.S.Jalal 200808 (BSI).

Dendrobium barbatulum Lindl., Gen. Sp. Orchid. P1.:

84. 1830. Fig. 4H.

FLOWERING: January-May.

LIFE FORM: Epiphyte.

HABITAT: Found in dry-deciduous, moist-deciduous and semi-evergreen forests at elevations between $50-1200 \mathrm{~m}$.

Distribution in Maharashtra: Kolhapur, Nandurbar, Nashik, Pune, Raigad, Ratnagiri, Satara, Sindhudurg and Thane.

General Distribution: India (Gujarat, Goa, Karnataka, Kerala, Maharashtra, Tamil Nadu), endemic to Western Ghats.

SPECimens EXAmined: Kolhapur district, Radhanagari, J.S.Jalal 200515 (BSI). Nandurbar district, Toranmal, J.S.Jalal 195109 (BSI). Nashik district, Igatpuri, H. Santapau 22957 (BLAT). Pune district, Bhimashankar, J.S.Jalal 200401 (BSI). Raigad district, Phanshad, J.S.Jalal 200885 (BSI). Ratnagiri district, Panchvali, Dapoli, J.S.Jalal 194943 (BSI). Satara district, Mahabaleshwar, J.S.Jalal 200811 (BSI). Sindhudurg district, Amboli, J.S.Jalal 195020 (BSI). Thane district, Tansa, J.S.Jalal 200863 (BSI).

Dendrobium crepidatum Lindl. \& Paxton, Paxton's

Fl. Gard. 1: 63. 1850. Fig. 5A.

FLowerIng: April-June.

LIFE FORM: Epiphyte.

НАвітAт: Found in semi-evergreen forests.
Distribution in Maharashtra: Kolhapur, Pune and Sindhudurg.

General Distribution: India (Assam, Manipur, Meghalaya, Mizoram, Nagaland, Arunachal Pradesh, Sikkim, West Bengal, Uttarakhand, Odisha, Goa, Karnataka, Kerala, Maharashtra, Tamil Nadu, Bihar, Jharkhand Chhattisgarh), Bangladesh, Bhutan, Nepal, China, Myanmar, Thailand, Laos, Vietnam.

Specimens eXamined: Kolhapur district, Ajara, M.M.Sardesai 1204 (SUK). Pune district, Bhovargiri, K.P.Janardhanan 76570 (BSI). Sindhudurg district, Shivapur, Dukanwad, B.G.Kulkarni 129647 (BSI).

Dendrobium herbaceum Lindl., Edward's, Bot. Reg. 26: Misc. 69. 1840. Fig. 4I.

Flowering: February-March.

LIFE FORM: Epiphyte.

HABITAT: Found in semi-evergreen and moist deciduous forests.

Distribution in MAHARAShtra: Ahmednagar, Kolhapur, Nashik, Pune, Satara, Sindhudurg and Thane.

General Distribution: India (Mizoram, West Bengal, Bihar, Chhattisgarh, Jharkhand, Madhya Pradesh, Andhra Pradesh, Odisha, Goa, Maharashtra, Karnataka, Kerala, Tamil $\mathrm{Nadu}$ ), endemic to India.

SPECimens EXAmined: Ahmednagar district, Piprichavran, Harishchandragadh, B.M.Wadhwa 127857 (BSI). Kolhapur district, Panhala, M.M.Sardesai 1825 (SUK). Nashik district, Ambewadi forest, P.L.Narasimhan 167517 (BSI). Pune district, Bhimashankar, J.S.Jalal 200723 (BSI). Satara district, Mahabaleshwar, J.S.Jalal 200413 (BSI). Sindhudurg district, Khamdadev plateau, J.S.Jalal 195016 (BSI). Thane: Tokavada range, K.V. Billore 115729 (BSI).

Dendrobium lawianum Lindl., J. Proc. Linn. Soc., Bot. 3: 10. 1858. Fig. 5B.

FLowerING: March-April; Leaves: May-October. LIFE FORM: Epiphyte.

Haвitat: Found in moist deciduous and semievergreen forests.

Distribution in Maharashtra: Kolhapur, Sindhudurg and Thane. 
General distribution: India (Goa, Karnataka, Kerala, Maharashtra, Tamil Nadu), endemic to Western Ghats.

SPecimens eXamined: Kolhapur district, Ajara, J.S.Jalal 200491 (BSI). Sindhudurg district, Amboli, J.S.Jalal 194963 (BSI). Thane district, Usgaon, N.Y.Das 3195 (BLAT).

Note: Govaerts et al. (2016), Tropicos (2016) and IPNI (2016) have treated this species as a synonym of Dendrobium crepidatum Lindl. However, Dendrobium lawianum can be distinguished based on characters such as, pseudobulbs curving in one direction, flowers white $c a .2 \mathrm{~cm}$ across, with obscure mentum, lip slightly clawed at the base without a yellow patch.

Dendrobium macrostachyum Lindl., Gen. \& Sp. Orchids P1.: 78. 1830. Fig. 5C.

FLowerING: May-June; Leaves: May-November.

LIFE FORM: Epiphyte.

HABITAT: Found in semi-evergreen forests.

Distribution in Maharashtra: Kolhapur, Pune, Satara and Sindhudurg.

General distribution: India (Arunachal Pradesh, West Bengal, Uttarakhand, Odisha, Goa, Karnataka, Maharashtra Jharkhand), Nepal, Bangladesh, Sri Lanka, Indonesia, Thailand, Vietnam.

SPeCimens EXAmined: Kolhapur district, Chandgad, J.S.Jalal 200436 (BSI). Pune district, Durga khilla, Hemadri 94362 (BSI). Ratnagiri district, Konal R.F., Bhedshi, B.G.Kulkarni 129422 (BSI). Satara district, Koyna valley, T.Cooke s.n. (BSI). Sindhudurg district, Danoli, S.M.Almeida 1649 (BLAT).

Dendrobium microbulbon A. Rich., Ann. Sci. Nat.

Bot. 2, 15: 19, t. 8. 1841. Fig. 5D.

Flowering: December-January; Leaves: JulyOctober.

LIFE FORM: Epiphyte.

HABITAT: Found in moist-deciduous forests and semi-evergreen forests.

Distribution in Maharashtra: Kolhapur, Nashik, Pune, Raigad, Satara, Sindhudurg and Thane.

General Distribution: India (Gujarat, Goa, Karnataka, Kerala, Maharashtra, Tamil Nadu), endemic to Western Ghats.
SPecimens EXAmined: Kolhapur: Udegiri, M.M.Sardesai 1492 (SUK). Pune: Junnar, J.S.Jalal 200818 (BSI). Nashik district, Igatpuri, Z.J.Kapadia 1374 (BLAT). Raigad district, Matheran, J.S.Jalal 200707 (BSI). Satara district, Mahabaleshwar, H.M.Chibber s.n. (BSI). Sindhudurg district, Amboli, J.S.Jalal 195021 (BSI). Thane district, Kasara, K.V.Billore 116451 (BSI).

Dendrobium nanum Hook.f., Hooker's Icon. Pl. 19: t. 1853. 1889. Fig. 5E.

Dendrobium mabelae Gammie, J. Bombay Nat. Hist. Soc. 16: 567. 1903.

FLowering: July-September.

LIFE FORM: Epiphyte.

Habitat: Found in semi-evergreen forest at elevations between 600-1200 m.

Distribution in Maharashtra: Raigad and Satara. General Distribution: India (Goa, Karnataka, Kerala, Maharashtra, Tamil Nadu), endemic to Western Ghats.

SPECIMENS EXAmined: Satara district, Mahabaleshwar, J.S.Jalal 200419 (BSI). Raigad district, Matheran, J.S.Jalal 200599 (BSI).

Dendrobium nodosum Dalzell, Hooker's J. Bot. Kew Gard. Misc. 4: 292. 1852.

Flickingeria nodosa (Dalzell) Seidenf., Dansk Bot. Ark. 34: 41. 1980.

Dendrobium macraei sensu Barbhuiya \& Salunkhe in Richardiana 15: 130. 2016.

FLOWERING: July-August.

LIFE FORM: Epiphyte.

HАвітAт: Found in semi-evergreen forests.

Distribution in Maharashtra: Kolhapur, Satara and Thane.

General Distribution: India (Goa, Karnataka, Kerala, Maharashtra, Tamil Nadu), endemic to Western Ghats.

Specimens eXamined: Kolhapur district, Chandgad, J.S.Jalal 195195 (BSI). Satara district, Mahadurga, Koyna, R.K.Kochhar 154704 (BSI). Thane district, Usgaon, N.Y.Das 3192 (BLAT).

Dendrobium ovatum (L.) Kraenzl. in H.G.A. Engler (Ed.), Pflanzenr., IV, 5 II B 21: 71. 1910. Fig. 5F.

Epidendrum ovatum L., Sp. P1.: 952. 1753.

LANKESTERIANA 18(1). 2018. (C) Universidad de Costa Rica, 2018. 
FLOWERING: September-February.

LIFE FORM: Epiphyte.

Habitat: Found in moist-deciduous and semievergreen forests.

Distribution in Maharashtra: Kolhapur, Mumbai Suburban, Satara, Sindhudurg, Raigad and Thane.

General Distribution: India (Andhra Pradesh, Karnataka, Gujarat, Goa, Kerala, Maharashtra, Tamil Nadu, Dadara \& Nagar Haveli), endemic to Western Ghats.

Specimens eXamined: Kolhapur district, Kalakdara, M.M.Sardesai 2033 (SUK). Mumbai Suburban district, Malad, G.L.Shah 9632 (BLAT). Raigad district, Matheran, N.A.Irani 2818 (BLAT). Satara district, Mahabaleshwar, J.S.Jalal 200805 (BSI). Sindhudurg district, Karulgaon, J.S.Jalal 200541 (BSI). Thane district, Murbad, J.S.Jalal 200730 (BSI).

Dendrobium peguanum Lindl., J. Proc. Linn. Soc., Bot. 3: 19. 1858. Fig. 5G.

Flowering: October-December; Leaves: JuneOctober.

LIFE FORM: Epiphyte.

Навітат: Found in dry-deciduous forests and moist-deciduous forests.

Distribution in Maharashtra: Kolhapur, Thane and Nashik.

General Distribution: India (Mizoram, Sikkim, West Bengal, Odisha, Gujarat, Karnataka, Maharashtra, Jharkhand, Chhattisgarh), Nepal, Myanmar, Thailand.

SPECIMENS EXAMINED: Kolhapur district, Amba ghat, J.S.Jalal 195035 (BSI). Nashik district, Igatpuri, Z.J.Kapadia 1375 (BLAT). Thane district, Kasara, Z.J.Kapadia 897 (BLAT).

\section{Epipogium Borkh.}

Epipogium roseum (D. Don) Lindl., J. Proc. Linn. Soc., Bot. 1: 177. 1857. Fig. 5H.

Limodorum roseum D. Don, Prodr. Fl. Nepal. 30. 1825. FLOWERING: May-June.

LIFE FORM: Mycoheterotrophic.

HaвiтAт: It prefers to grow under the dense semievergreen forests.

Distribution IN Maharashtra: Kolhapur and Satara.
General Distribution: India (Meghalaya, Mizoram, Arunachal Pradesh, Sikkim, West Bengal, Jammu \& Kashmir, Himachal Pradesh, Uttarakhand, Karnataka, Kerala, Maharashtra, Tamil Nadu), Nepal, Sri Lanka, China, Myanmar, Indonesia, Japan, Malaysia, Philippines, Thailand, Laos, Vietnam, Pacific islands, Africa.

Specimens eXamined: Kolhapur district, AmbaVishalgad road, M.M.Sardesai 2035 (SUK). Satara district, Dicholi, Koyna, Bachulkar 3502 (SUK).

Eulophia R.Br. ex Lindl.

Eulophia dabia (D.Don) Hochr., Bull. New York Bot. Gard.6: 270. 1910.

Bletia dabia D.Don, Prodr. Fl. Nepal.: 30. 1825.

FLOWERING: March - April.

LIFE FORM: Terrestrial.

HABiтAT: Found in dry-deciduous forests near marshy and grassy area along river-beds.

Distribution in Maharashtra: Nagpur.

General Distribution: India (Assam, Manipur, Arunachal Pradesh, Sikkim, West Bengal, Himachal Pradesh, Jammu \& Kashmir, Uttarakhand, Odisha, Maharashtra, Bihar, Haryana, Jharkhand, Punjab, Uttar Pradesh, Andaman \& Nicobar), Bangladesh, Bhutan, Nepal, Pakistan, Afganistan, China, Myanmar, Tajikistan, Turkmenistan, Uzbekistan.

Note: This species is included based on the authority of Ugemuge (1986) from Nagpur. He collected it from Ambazari. His collection number 1010 couldn't be traced in the Herbarium, Nagpur University, Nagpur.

Eulophia epidendraea (Retz.) C.E.C.Fisch. in J.S.Gamble, Fl. Madras: 1434. 1928. Serapias epidendraea Retz., Observ. Bot. 6: 65. 1791.

FLowering: November-January.

LIFE FORM: Terrestrial.

НавітAт: Found under the shade of moistdeciduous forests and dry deciduous forests between 600-800 m.

Distribution in Maharashtra: Pune and Sindhudurg. General Distribution: India (West Bengal, Uttarakhand, Odisha, Andhra Pradesh, Karnataka, Kerala, Maharashtra, Tamil 


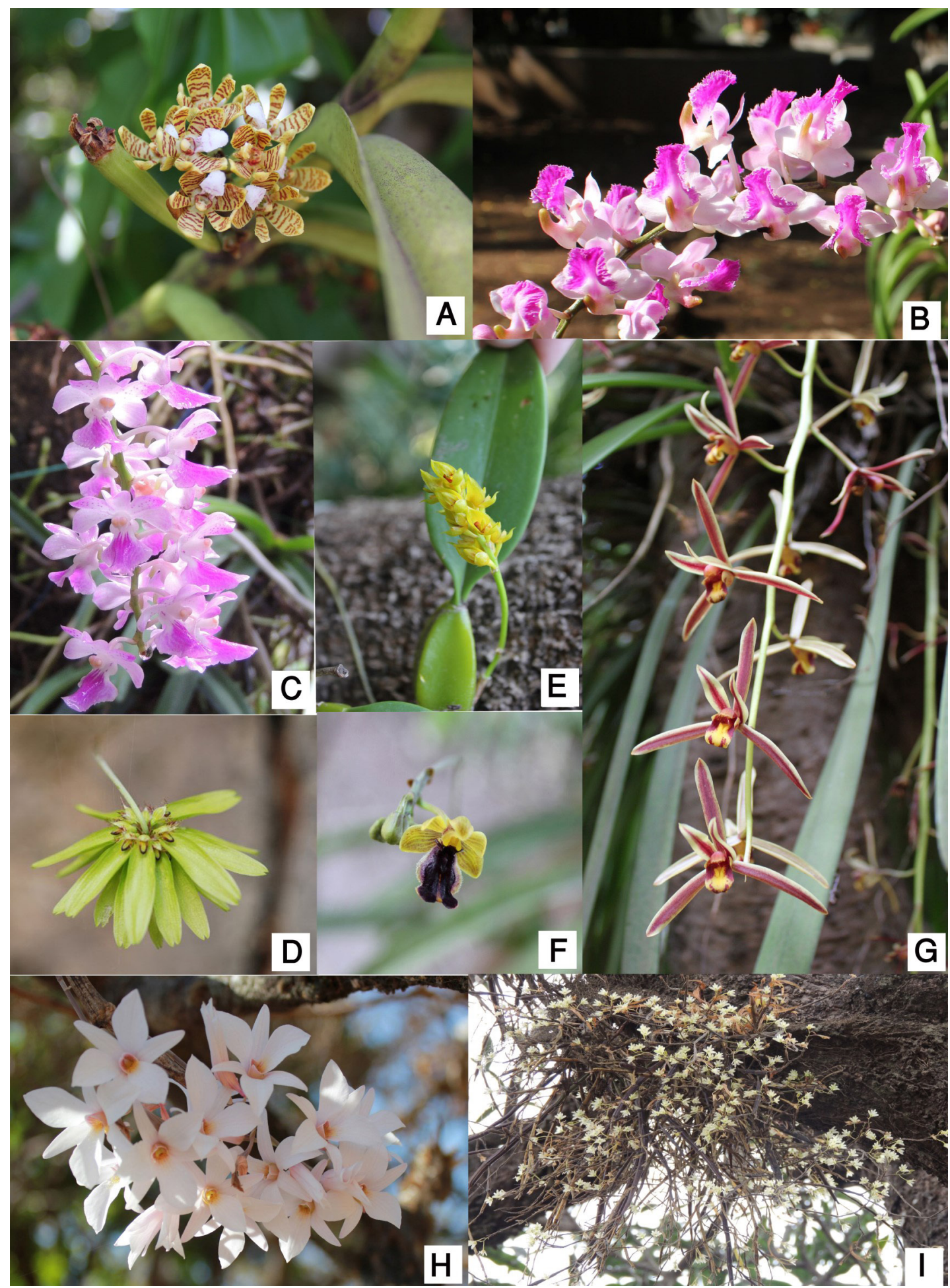

Figure 4. A. Acampe praemorsa, B. Aerides crispa, C. Aerides maculosa, D. Bulbophyllum fimbriatum, E. Bulbophyllum sterile, F. Cottonia peduncularis, G. Cymbidium bicolor, H. Dendrobium barbatulum, I. Dendrobium herbaceum 


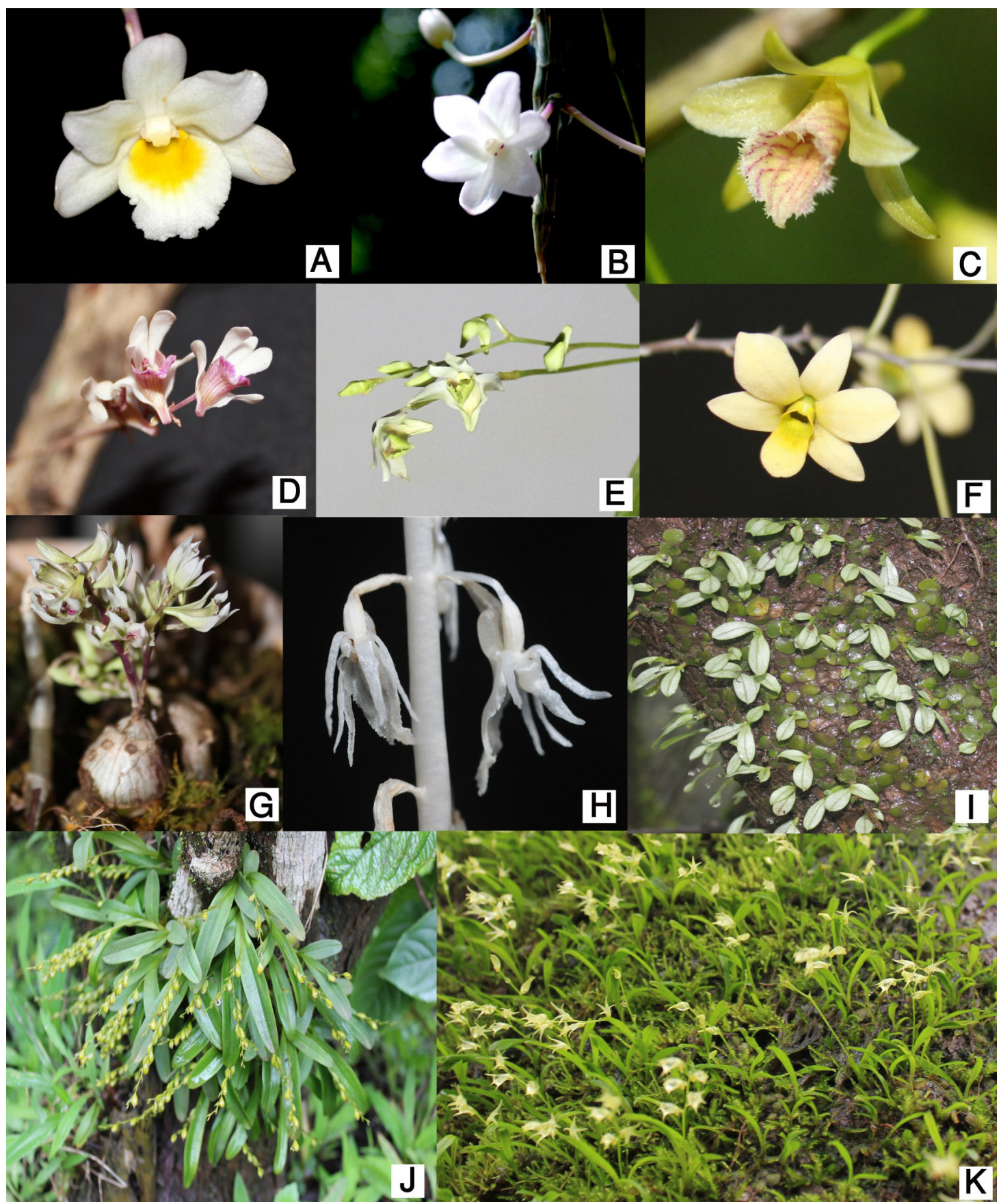

Figure 5. A. Dendrobium crepidatum, B. Dendrobium lawianum, C. Dendrobium macrostachyum, D. Dendrobium microbulbon, E. Dendrobium nanum, F. Dendrobium ovatum, G. Dendrobium peguanum, H. Epipogium roseum, I. Conchidium exile, J. Conchidium filiforme, K. Conchidium microchilos.

Nadu, Chhattisgarh, Andaman \& Nicobar), Bangladesh, Sri Lanka.

Specimens eXAmined: Pune district, Khandala,
H.Santapau 10532 (BLAT). Sindhudurg district, Ramghat, B.G.Kulkarni 119300 A (BSI). 
Eulophia graminea Lindl., Gen. Sp. Orchid. P1.: 182. 1833. Fig. 6B.

FLOWERING: March-April.

LIFE FORM: Terrestrial.

HABITAT: Found in moist-deciduous forests, scrub forests, in open places at $c .600 \mathrm{~m}$.

Distribution in Maharashtra: Gadchiroli, Osmanabad and Sangli.

General Distribution: India (Assam, Meghalaya, Mizoram, Nagaland, Tripura, Arunachal, Pradesh, Sikkim, West Bengal, Uttarakhand, Andhra Pradesh, Odisha, Kerala, Maharashtra, Tamil Nadu, Bihar, Jharkhand, Madhya Pradesh, Punjab, Uttar Pradesh, Andaman \& Nicobar), Bangladesh, Bhutan, Nepal, Sri Lanka, China, Myanmar, Indonesia, Japan, Malaysia, Singapore, Thailand, Laos, Vietnam.

SPecimens EXAmined: Gadchiroli district, Podewada, R.S.Govekar 1009 (BAMU). Osmanabad district, Apsinga, R.D.Gore 78 (Walchand College of Arts and Science, Solapur). Sangli district, Sangli, S.R. Yadav 4397 (SUK).

Eulophia herbacea Lindl., Gen. Sp. Orchid. P1. 182. 1833. Fig. 6C.

FLOWERING: June-July.

LIFE FORM: Terrestrial.

HABIтAT: Found under the shade of moist-deciduous forests and in open scrub area between 200$600 \mathrm{~m}$.

Distribution in Maharashtra: Mumbai Suburban and Thane.

General distribution: India (Manipur, Mizoram, Tripura, Sikkim, West Bengal, Himanchal Pradesh, Uttarakhand, Odisha, Karnataka, Gujarat, Maharashtra, Chhattisgarh, Madhya Pradesh), Bangladesh, Nepal, China, Myanmar, Thailand, Laos.

Specimens examined: Mumbai Suburban district, Borivali National Park, Z.J.Kapadia 1252 (BLAT). Pune district, Lonavala, S.R. Yadav s.n. (SUK). Thane district, Mumbra, H.Santapau 15664 (BLAT).

Eulophia ochreata Lindl., J. Proc. Linn. Soc., Bot. 3: 24. 1858.

FLOWERING: June-July.

LIFE FORM: Terrestrial.
HABITAT: Found in slopes and rocky areas of dry-deciduous and moist-deciduous forests between 100-1000 m.

Distribution IN MAHARAShTRA: Amravati, Chandrapur, Kolhapur, Mumbai Suburban, Nandurbar, Pune, Ratnagiri and Thane.

General Distribution: India (Meghalaya, West Bengal, Odisha, Andhra Pradesh, Gujarat, Karnataka, Maharashtra, Chhattisgarh, Jharkhand, Madhya Pradesh, Rajasthan), endemic to India.

Specimens examined: Amravati district, Melghat, M.Y.Ansari 147182 (BSI). Chandrapur district, Devada forest, B.M.Wadhwa 137434 A (BSI). Kolhapur district, Panhala, M.M.Sardesai 1522 (SUK). Mumbai Suburban district, Mumbai, H.Santapau 1266 (BLAT). Nandurbar district, Toranmal, J.S.Jalal 195129 (BSI). Pune district, Sinhgad, M.Y.Ansari 87371 (BSI). Ratnagiri district, Malwan, B.G.Kulkarni 131787 (BSI). Thane district, Bhivandi range, K.V.Billore 116239 (BSI).

Eulophia pratensis Lindl., J. Proc. Linn. Soc., Bot. 3: 25. 1858. Fig. 6D.

Eulophia ramentacea Wight, Icon. Pl. Ind. Orient. 5: t. 1666. 1851, nom. illeg.

FLOWERING: December-February.

LIFE FORM: Terrestrial.

НАвітAт: Found in marshy and grassy area along river-beds and seasonal water channels between $500-1000 \mathrm{~m}$.

Distribution in Maharashtra: Kolhapur, Pune and Satara.

General Distribution: India (Gujarat, Karnataka, Kerala, Maharashtra, Tamil Nadu), endemic to Peninsular India.

SPECIMENS EXAmined: Kolhapur district, Radhanagari, V.D.Patil 75 (SUK). Pune district, Pashan, G.A.Gammie s.n. (BSI). Satara district, Khindwadi, M.P.Bachulkar 5424 (SUK).

Eulophia spectabilis (Dennst.) Suresh in D.H.Nicolson, C.R.Suresh \& K.S.Manilal, Interpret. Van Rheede's Hort. Malab.: 300. 1988. Fig. 6E.

Wolfia spectabilis Dennst., Schlüssel Hortus Malab.: 38. 1818.

FLOWERING: It produces floral stalks on the onset of rain from May to June and foliar shoot emerges 
after flowering shoot.

LIFE FORM: Terrestrial.

Haвitat: Found in dry-deciduous forests and occasionally along the edges of semi-evergreen forests at elevations between $50-1250 \mathrm{~m}$.

Distribution in Maharashtra: Kolhapur, Pune, Satara and Sindhudurg.

General distribution: India (Assam, Manipur, Meghalaya, Mizoram, Nagaland, Tripura, Arunachal Pradesh, Sikkim, West Bengal, Uttarakhand, Uttar Pradesh, Jharkhand, Bihar, Madhya Pradesh, Chhattisgarh, Punjab, Odisha, Andhra Pradesh, Karnataka, Maharashtra, Kerala, Tamil Nadu, Andaman \& Nicobar), Bangladesh, Bhutan, Nepal, Sri Lanka, China, Myanmar, Japan, Indonesia, Malaysia, Philippines, Thailand, Singapore, Indo-China, New Guinea, Pacific islands.

Specimens EXAmined: Kolhapur district, Radhanagari, J.S.Jalal 200521 (BSI). Pune district, Lavasa, J.S.Jalal 195106 (BSI). Satara district, Mahabaleshwar, J.S.Jalal 200424 (BSI). Sindhudurg district, Amboli Ghat, J.S.Jalal 200473 (BSI).

\section{Gastrochilus D.Don}

Gastrochilus flabelliformis (Blatt. \& McCann) C.J. Saldanha in C.J. Saldanha \& Nicolson, Fl. Hassan Dist. 830. 1976.

Saccolabium flabelliforme Blatt. \& McCann in J. Bombay Nat. Hist. Soc. 35: 722, t. 1. 1932.

FLOWERING: Mach-July.

LIFE FORM: Epiphyte.

Habit and Habitat: Epiphytic. Found in evergreen forests ca $400 \mathrm{~m}$.

Distribution in Maharashtra: Sindhudurg.

General Distribution: India (Maharashta, Goa, Karnataka, Kerala), endemic to Western Ghats.

Note: This was reported by S. M. Almeida (1990) as Gastrochilus calceolaris from Sawantwadi. There are no herbarium records of its collection in BLAT. Sardesai et al. (2002) reported it from Dodamarg in Sindhudurg district. During herbarium scrutiny his collection number 2502 (SUK) couldn't be traced.
Geodorum G. Jacks.

Geodorum densiflorum (Lam.) Schltr.in Fedde, Repert. 4: 259. 1919. Fig. 6F.

Limodorum densiflorum Lam., Encycl. 3: 516. 1792. FLOWERING: June-July.

LIFE FORM: Terrestrial.

HabitaT: Found under the shade of semi-evergreen and moist-deciduous forests between $400-800 \mathrm{~m}$.

Distribution IN MAHARAshtra: Gadchiroli, Kolhapur and Nashik.

General Distribution: India (Assam, Manipur, Meghalaya, Mizoram, Nagaland, Tripura, Arunachal Pradesh, Sikkim, West Bengal, Uttarakhand, Andhra Pradesh, Odisha, Goa, Karnataka, Kerala, Maharashtra, Tamil Nadu, Bihar, Chhattisgarh, Jharkhand, Madhya Pradesh, Andaman \& Nicobar), Bangladesh, Bhutan, Nepal, Sri Lanka, China, Myanmar, Indonesia, Japan, Malaysia, Philippines, New Guinea, Thailand, Indo-China, S.E.Asia, Pacific Islands, Australia.

SPECIMENS EXAMINED: Gadchiroli district, Surajgad, R.S.Govekar 21 (BAMU). Kolhapur district, Chandgad, J.S.Jalal 195170 (BSI). Nashik district, Satana, P.L.Narasimhan 167662 (BSI).

Geodorum recurvum (Roxb.) Alston in H.Trimen, Handb. Fl. Ceylon 6(Suppl.): 276.1931. Limodorum recurvum Roxb., P1. Coromandel 1: t. 39. 1795.

Flowering: June-July.

LIFE FORM: Terrestrial.

HABITAT: Found under the shade of moist-deciduous forests.

Distribution in Maharashtra: Gadchiroli.

General distribution: India (Assam, Manipur,

Andhra Pradesh, Odisha, Jharkhand, Maharashtra), Sri Lanka, China, Myanmar, Thailand, Cambodia, Vietnam.

SPECIMEN EXAMINED: Gadchiroli district, Jharapapada, R.S.Govekar 1667 (BAMU).

\section{Habenaria Willd.}

Habenaria brachyphylla (Lind1.) Aitch., J. Linn. Soc., Bot. 19: 188. 1882. Fig. 6G.

Platanthera brachyphylla Lindl., Gen. Sp. Orchid. P1.: 293. 1835. 
Habenaria crassifolia A.Rich., Ann. Sci. Nat., Bot., II, 15: 72. 1841.

Flowering: August-October.

LIFE FORM: Terrestrial.

HавітAт: Found in semi-evergreen forests along the sedges and open grassy slopes at higher plateaus between 200-1350 m.

Distribution in MAHARAshtra: Bhandara, Kolhapur, Nashik, Pune and Satara.

General Distribution: India (Odisha, Karnataka, Kerala, Maharashtra, Tamil Nadu), endemic to Peninsular India.

Specimens examined: Bhandara district, Pangni forest, S.K.Malhotra 144573 (BSI). Kolhapur district, Panhala, D.H.Jugadale s.n. (SUK). Nashik district, Ambewadi, P.L.Narasimhan $167518 \mathrm{~A}$ (BSI). Pune district, Ambe ghat, J.S.Jalal 200815 (BSI). Satara district, Mahabaleshwar, J.S.Jalal 200423 (BSI).

Habenaria commelinifolia (Roxb.) Wall. ex Lindl., Gen. Sp. Orchid. P1. 325. 1835. Fig. 6H.

Orchis commelinifolia Roxb., Fl. Ind. ed. 1832, 3: 451. 1832.

FLowering: August-November.

LIFE FORM: Terrestrial.

Haвitat: Found in open grassy slopes between dry deciduous and semi-evergreen forests between 0 and $600 \mathrm{~m}$.

Distribution in Maharashtra: Gondia, Mumbai Suburban, Pune, Raigad, Ratnagiri, Sindhudurg and Thane.

General Distribution: India (Manipur, Sikkim, West Bengal, Himachal Pradesh, Jammu \& Kashmir, Uttarakhand, Odisha, Karnataka, Kerala, Maharashtra, Bihar, Chhattisgarh, Jharkhand, Madhya Pradesh, Punjab, Uttar Pradesh), Nepal, Myanmar, Thailand, Vietnam.

Specimens eXamined: Gondia district, Tower road, D.N.Patil 179412 (BSI). Mumbai Suburban district, Andheri, H.Santapau 7372 (BLAT). Pune district, Khandala, Z.J.Kapadia 1509 (BLAT). Raigad district, Matheran, N.A.Irani 4565 (BLAT). Ratnagiri district, Dapoli, J.S.Jalal 194946 (BSI). Sindhudurg district, Insuli, S.M.Almeida 3926 (BLAT). Thane district, Mumbra, K.V.Shenoy 4299 (BLAT).
Habenaria crinifera Lindl., Gen. Sp. Orchid. Pl. 323. 1835. Fig. 6I.

FLOWERING: July-August.

LIFE FORM: Terrestrial.

НАвітAт: Found in semi-evergreen and evergreen forests between $600-800 \mathrm{~m}$.

Distribution in Maharashtra: Kolhapur and Satara.

General Distribution: India (Goa, Karnataka, Kerala, Maharashtra, Tamil Nadu), Sri Lanka.

Specimens eXamined: Kolhapur district, Chandgad, J.S.Jalal 195200 (BSI). Satara district, Koyna valley, Z.J.Kapadia 2893 (BLAT).

Habenaria digitata Lindl., Gen. \& Sp. Orchid. P1. 307. 1835. Fig. 6J.

Flowering: July-September.

LIFE FORM: Terrestrial.

HABIтAT: Found under the shade of moist deciduous and semi-evergreen forests between 400-800 m.

Distribution in Maharashtra: Akola, Kolhapur, Nashik, Pune, Sindhudurg and Thane.

General Distribution: India (Assam, Arunachal Pradesh, Sikkim, West Bengal, Himachal Pradesh, Uttarakhand, Andhra Pradesh, Odisha, Gujarat, Karnataka, Kerala, Maharashtra, Tamil Nadu, Jharkhand, Chhattisgarh, Madhya Pradesh, Uttar Pradesh, Rajasthan), Bangladesh, Nepal, Pakistan, Myanmar, Cambodia, Laos.

Specimens eXamined: Akola district, Narnala fort, S.Y.Kamble 154073A (BSI). Kolhapur district, Radhanagari, 197733 (BSI). Nashik district, Markandey hill, R.D.Pataskar 108822 (BSI). Pune district, Rajmachi, J.S.Jalal 200576 (BSI). Sindhudurg district, Amboli, J.S.Jalal 194949 (BSI). Thane district, Malshej Ghat, J.S.Jalal 200847 (BSI).

Habenaria diphylla (Nimmo) Dalzell in Hooker's, J. Bot. Kew Gard. Misc. 2: 262. 1850. Fig. 6K. Liparis diphyllos Nimmo in J.Graham, Cat. P1. Bombay: 252.1839.

FlOWERING: August-October.

LIFE FORM: Terrestrial.

Habit and Habitat: Terrestrials, found in lateritic plateaus growing along with grasses and herbs at lower elevations with clayey soil between 50-700 m. 
Distribution in Maharashtra: Kolhapur, Nashik and Sindhudurg.

General distribution: India (Meghalaya, Sikkim, West Bengal, Himachal Pradesh, Uttarakhand, Odisha, Andhra Pradesh, Goa, Karnataka, Kerala, Maharashtra, Tamil Nadu, Bihar, Jharkhand, Chhattisgarh), Bangladesh, Bhutan, Nepal, China, Myanmar, Philippines, Thailand.

SPECimEns EXAmined: Kolhapur district, Chandgad, J.S.Jalal 197740 (BSI). Nashik district, Igatpuri, J.S.Jalal 200871 (BSI). Sindhudurg district, Malvan, J.S.Jalal 200468 (BSI).

Habenaria elwesii Hook.f., Bot. Mag. 122: t. 7478. 1896.

Flowering: September-October.

LIFE FORM: Terrestrial.

Habit and Habitat: Terrestrials, found in edges of semi-evergreen forests at elevation $800 \mathrm{~m}$.

Distribution in Maharashtra: Kolhapur.

General Distribution: India (Maharashta, Karnataka, Kerala and Tamil Nadu), endemic to Western Ghats.

Note: This species is included based on Bachulkar (2012) who reported it from Kolik in Kolhapur district. The specimen number 20349 deposited at SUK herbarium couldn't be traced.

Habenaria foliosa A. Rich., Ann. Sci. Nat., Bot., 2, 15: 71, t. 3 A. 1841. Fig. 6L.

Habenaria foliosa var. foetida (Blatt. \& McCann) Bennet, J. Econ. Taxon. Bot. 5: 452. 1984.

FLOWERING: July-August.

LIFE FORM: Terrestrial.

Haвitat: Found under the shade and edges of semievergreen forests between 400-800 m.

Distribution in Maharashtra: Gadchiroli, Kolhapur, Nashik, Pune, Raigad, Satara and Thane.

General distribution: India (West Bengal, Goa, Gujarat, Karnataka, Kerala, Maharashtra, Tamil Nadu, Odisha, Chhattisgarh, Madhya Pradesh), endemic to Western Ghats.

SPecimens EXAmined: Gadchiroli district, R.S.Govekar 0487 (BAMU). Kolhapur district, Radhanagari, M.M.Sardesai 1455 (SUK). Nashik district, Kasara ghat, J.S.Jalal 200869 (BSI). Pune district, Nane ghat, J.S.Jalal 200840 (BSI). Raigad district, Matheran,
N.A.Irani 5036 (BLAT). Satara district, Koyna, Prajakta Pathare 198885 (BSI). Thane district, Ghodabunder, Z.J.Kapadia 2103 (BLAT).

Habenaria furcifera Lindl., Gen. \& Sp. Orchid Pl.: 319. 1835. Fig. 6M.

Flowering: August-September.

LIFE FORM: Terrestrial.

HABITAT: Found under the shade of semi-evergreen forests and dry deciduous forests between 400 $900 \mathrm{~m}$.

Distribution in Maharashtra: Ahmednagar, Amravati, Kolhapur, Nashik and Sindhudurg.

General distribution: India (Assam, Nagaland, Arunachal Pradesh, Sikkim, West Bengal, Himachal Pradesh, Jammu \& Kashmir, Uttarakhand, Kerala, Madhya Pradesh, Odisha, Andhra Pradesh, Tamil Nadu, Maharashtra, Gujarat, Bihar, Jharkhand, Chhattisgarh, Punjab, Uttar Pradesh), Bangladesh, Bhutan, Nepal, China, Myanmar, Thailand.

SPeCimens eXAmined: Ahmednagar district, Waranghushi forest, B.M.Wadhwa 128357 (BSI). Amravati district, Semadoh, M.Y.Ansari 149399 (BSI). Kolhapur district, Kondoshi, M.M.Sardesai 1553 (SUK). Nashik district, base of Markenday hill, 21.07.2014, J.S.Jalal 195146 (BSI). Sindhudurg district, Amboli, S.M.Almeida 5022 (BLAT).

Habenaria gibsonii Hook.f., Fl. Brit. India 6: 135. 1890. Fig. 7A.

Habenaria digitata var. gibsonii (Hook.f.) C.E.C.Fisch. in J.S.Gamble, Fl. Madras: 1469. 1928.

Habenaria gibsonii var. foliosa (A.Rich) Santapau \& Kapadia, J. Bombay Nat. Hist. Soc. 56: 194. 1959.

Habenaria gibsonii var. foetida Blatt. \& McCann,

J. Bombay Nat. Hist. Soc. 36: 16. 1932.

Habenaria foliosa var. gibsonii (Hook.f.)

Bennet, J. Econ. Taxon. Bot. 5: 452. 1984.

FLowering: July-August.

LIFE FORM: Terrestrial.

HaвiтAT: Found under the shade and edges of semievergreen forests between $300-800 \mathrm{~m}$.

Distribution in Maharashtra: Akola, Kolhapur, Nandurbar, Nashik, Pune, Raigad, Satara and Thane. 
General Distribution: India (Gujarat, Karnataka, Maharashtra, Odisha, Chhattisgarh, Madhya Pradesh), endemic to Peninsular India.

SPecimens eXAmined: Akola district, Narnala fort, S.Y.Kamble 150456 (BSI). Kolhapur district, Tilari, J.S.Jalal 197714 (BSI). Nandurbar district, Toranmal, J.S.Jalal 195122 (BSI). Nashik district, base of Markendey hill, J.S.Jalal 195143 (BSI). Pune district, Khandala, B.M.Wadhwa 109972 (BSI). Raigad district, Matheran, B.M.Wadhwa 109746 (BSI). Satara district, Mahabaleshwar, B.M.Wadhwa 109642 (BSI). Thane district, Mumbra hill, K.V.Billore 116608 (BSI).

Habenaria grandifloriformis Blatt. \& McCann, J. Bombay Nat. Hist. Soc. 36: 17. 1932. Fig. 7B.

FLowering: May-July.

LIFE FORM: Terrestrial.

НАвітAт: Found in lateritic plateaus, mountain grassy slopes at elevation between $100 \mathrm{~m}$ and $1200 \mathrm{~m}$.

Distribution in Maharashtra: Akola, Kolhapur, Nashik, Pune, Raigad, Ratnagiri, Satara, Sindhudurg and Thane.

General distribution: India (Assam, Chhattisgarh, Odisha, Gujarat, Maharashtra, Karnataka, Kerala and Tamil Nadu), endemic to Peninsular India.

SPecimens EXAmined: Akola district, Narnala fort, S.Y.Kamble 150473 (BSI). Kolhapur district, Radhanagari, J.S.Jalal 200534 (BSI). Nashik district, Saturli, J.S.Jalal 195150 (BSI). Pune district, Nane ghat, J.S.Jalal 200835 (BSI). Raigad district, Karjat, G.L.Shah 10771 (BLAT). Ratnagiri district, Rajapur, J.S.Jalal 200463 (BSI). Satara district, Mahabaleshwar, J.S.Jalal 200418 (BSI). Sindhudurg district, Sawantwadi, J.S.Jalal 200472 (BSI). Thane district, Bhiwandi range, K.V.Billore 116241 (BSI).

Habenaria heyneana Lindl., Gen. Sp. Orchid. P1. 320.

1835. Fig. 7C.

FLOWERING: July-September.

LIFE FORM: Terrestrial.

Habit And Habitat: Terrestrials, found in lateritic plateaus and open mountain grassy slopes between $500-1200 \mathrm{~m}$.
Distribution in Maharashtra: Kolhapur, Nashik, Pune, Satara and Sindhudurg.

General distribution: India (Andhra Pradesh, Goa, Karnataka, Kerala, Maharashtra, Tamil $\mathrm{Nadu}$ ), endemic to Western Ghats.

SPECIMENS EXAmined: Kolhapur district, Radhanagari, J.S.Jalal 200533 (BSI). Nashik district, Aryaneri hill, P.L.Narasimhan 165117 (BSI). Pune district, Karla caves, J.S.Jalal 200547 (BSI). Satara district, Panchgani, J.S.Jalal 200445 (BSI). Sindhudurg district, Amboli, J.S.Jalal 194951 (BSI).

Habenaria hollandiana Santapau, F1. Purandar 126. 1957.

Habenaria affinis Wight, Icon. Pl. Ind. Orient. 5: t. 1707, 1851, nom. illeg.

FLowering: September-October.

LIFE FORM: Terrestrial.

HАвітAт: Found in grassy slopes c. $600 \mathrm{~m}$.

Distribution in Maharashtra: Pune.

General Distribution: India (Maharashtra and Karnataka), endemic to Western Ghats.

NotE: This is a rare orchid in Maharashtra and reported by Woodrow from Purandhar. No subsequent botanist after Woodrow's has been able to locate this plant in Maharashtra. This species has not been found during the recent exploration also.

Habenaria longicorniculata J. Graham, Cat. Bombay, Pl. 202. 1839. Fig. 7D.

FLowering: July-December.

LIFE FORM: Terrestrial.

Habit and Habitat: Terrestrials, found in lateritic plateaus and grassy slopes between 100-1200 m.

Distribution in Maharashtra: Akola, Aurangabad, Kolhapur, Nashik, Pune, Raigad, Satara, Sindhudurg and Thane.

General distribution: India (Andhra Pradesh, Odisha, Goa, Gujarat, Karnataka, Kerala, Maharashtra, Tamil Nadu, Jharkhand, Chhattisgarh, Madhya Pradesh, Rajasthan), Sri Lanka.

Specimens EXAmined: Akola district, Narnala fort, S.Y.Kamble 150475 (BSI). Aurangabad district, Mhaigmal hill, K.P.Janardhanan 100079 (BSI). Kolhapur district, Gabighat, 
J.S.Jalal 200546 (BSI). Nashik district, Igatpuri, J.S.Jalal 200870 (BSI). Pune district, Khandala, J.S.Jalal 200551 (BSI). Raigad district, Matheran, J.S.Jalal 200582 (BSI). Satara district, Kas plateau, M.P.Bachulkar 5201 (SUK). Sindhudurg district, Amboli, J.S.Jalal 194953 (BSI). Thane district, Malshej Ghat, J.S.Jalal 200845 (BSI).

Habenaria marginata Colebr. in Hook., Exot. Fl. 2, 17: t. 136. 1824. Fig. 7E.

Habenaria marginata var. flavescens (Hook. f.) T. Cooke, Fl. Bombay 2: 721. 1907.

Flowering: August-October.

LIFE FORM: Terrestrial.

Haвitat: Found under the shade of bushes in moist deciduous forests and also in open grasslands up to $1200 \mathrm{~m}$.

Distribution IN MAharashtra: Ahmednagar, Amravati, Aurangabad, Bhandara, Chandrapur, Gadchiroli, Kolhapur, Mumbai, Nagpur, Nashik, Osmanabad, Pune, Raigad, Ratnagiri, Satara and Sindhudurg.

General distribution: India (Meghalaya, West Bengal, Himachal Pradesh, Jammu \& Kashmir, Uttarakhand, Odisha, Andhra Pradesh, Daman \& Diu, Goa, Gujarat, Karnataka, Kerala, Maharashtra, Tamil Nadu, Dadara \& Nagar Haveli, Jharkhand, Chhattisgarh, Madhya Pradesh, Punjab, Rajasthan), Bangladesh, Bhutan, Nepal, China, Myanmar, Thailand.

SPecimens EXAmined: Kolhapur district, Gaganbawda, B.G.Chowgule 6(SUK). Mumbai Suburban district, Andheri, H.Santapau 23323 (BLAT). Nashik district, Katipada, P.L.Narasimhan 167623 (BSI). Pune district, Khadakvasala, R.S.Raghavan 64285 (BSI). Raigad district, Chakachaamaal, Phansad, J.S.Jalal 20088 (BSI). Ratnagiri district, Dapoli, J.S.Jalal 194947 (BSI). Satara district, Yavteshwar, M.P.Bachulkar 5200 (SUK). Sindhudurg district, Vengurla, D.H.Jagdale s.n. (SUK).

Habenaria multicaudata Sedgw., Rec. Bot. Surv. India 6: 352. 1919. Fig. 7F.

Flowering: August-October.

LIFE FORM: Terrestrial.

Habitat: Found under the shade of semi-evergreen forests at elevations between 700-1000 m.

Distribution in MAHARASHTRA: Akola and Kolhapur.

General Distribution: India (Maharashtra, Goa, Karnataka, Kerala, Tamil Nadu), endemic to Western Ghats.

Specimens eXamined: Akola district, Narnala fort, S.Y.Kamble 150973A (BSI). Kolhapur district, Chandgad, J.S.Jalal 195181 (BSI).

Habenaria ovalifolia Wight, Icon. P1. Ind. Orient. 5: t. 1708. 1851. Fig. 7G.

FLOWERING: August-October.

LIFE FORM: Terrestrial.

Haвitat: Found under the shade localities and forest edges of semi-evergreen forests between 400-1200 m.

Distribution in Maharashtra: Kolhapur, Mumbai Suburban, Pune, Raigad, Satara and Thane.

General distribution: India (Karnataka, Kerala, Maharashtra, Tamil Nadu), endemic to Western Ghats.

Specimens eXamined: Kolhapur district, Chandgad, J.S.Jalal 194985 (BSI). Mumbai Suburban district, Borivali, Z.J.Kapadia 1273 (BLAT). Pune district, Khandala, J.S.Jalal 200549 (BSI). Raigad district, Phansad, J.S.Jalal 200878 (BSI). Satara district, Mahabaleshwar, S.R.Yadav 5792 (SUK). Thane district, Malshej Ghat, J.S.Jalal 200849 (BSI).

Habenaria panigrahiana S.Misra, Blumea 27: 213.1981

Flowering: August-October.

LIFE FORM: Terrestrial.

HABITAT: Found under the shade moist deciduous forests.

Distribution in Maharashtra: Gadchiroli.

General Distribution: India (Andhra Pradesh, Maharashtra, Odisha, Tamil Nadu), endemic to Peninsular India.

SPECimen eXAmined: Gadchiroli district, Amdeli, 09.11.2010, R.S.Govekar 623 (BAMU).

Habenaria plantaginea Lindl., Gen. \& Sp. Orchid. Pl.: 323. 1835. Fig. 7H.

Flowering: August- October.

LIFE FORM: Terrestrial.

HaвiтAт: Found under shade of semi-evergreen forests between 100-1200 m. 
Distribution IN Maharashtra: Ahmednagar, Bhandara, Kolhapur, Nashik, Pune, Raigad and Sindhudurg.

General distribution: India (Assam, Meghalaya, Arunachal Pradesh, Sikkim, West Bengal, Himachal Pradesh, Jammu \& Kashmir, Uttarakhand, Andhra Pradesh, Odisha, Karnataka, Kerala, Maharashtra, Tamil Nadu, Chhattisgarh, Jharkhand, Madhya Pradesh, Punjab, Haryana, Uttar Pradesh), Nepal, Sri Lanka, Japan, Vietnam.

SPECIMENS EXAMINED: Ahmednagar district, Harischchandragad, B.M.Wadhwa 127856 (BSI). Bhandara district, Pikagheri forest, S.K.Malhotra 144880 (BSI). Kolhapur district, Patgaon, M.M.Sardesai 199 (SUK). Nashik district, Sawarna, John Cherian 112788 (BSI). Pune district, Bhimashankar, K.P.Janardhanan 81809 (BSI). Raigad district, Matheran, J.S.Jalal 200710 (BSI). Satara district, Mahabaleshwar, P.V.Bole 1200 (BLAT). Sindhudurg district, Amboli Ghat, R.S.Rao 131568A (BSI).

Habenaria rariflora A. Rich., Ann. Sci. Nat., Bot. 2, 15: 70, t. 2 D. 1841. Fig. 7I.

FLOWERING: July-September.

LIFE FORM: Terrestrial.

Habitat: Found in higher plateaus and moist rocks near seasonal waterfall between 500-1200 m.

Distribution IN MAharashtra: Ahmednagar, Kolhapur, Pune, Raigad, Satara and Sindhudurg.

General distribution: India (Andhra Pradesh, Karnataka, Kerala, Maharashtra, Tamil Nadu), endemic to Western Ghats.

SPECIMENS EXAMINED: Ahmednagar district, Harischandragarh, J.S.Jalal s.n. (BSI). Kolhapur district, Gaganbavada ghat, J.S.Jalal 200544 (BSI). Pune district, Ambe ghat, Junnar, J.S.Jalal 200817 (BSI). Raigad district, Matheran, J.S.Jalal 200592 (BSI). Satara district, Pasarni ghat, J.S.Jalal 200446 (BSI). Sindhudurg district, Amboli, J.S.Jalal 200474 (BSI).

Habenaria roxburghii Nicolson in C.J. Saldanha \& Nicolson, Fl. Hassan Dist. 834. 1976. Fig. 7J.

Orchis plantaginea Roxb., Pl. Coromandel 1: 32, t.37. 1795.
Habenaria platyphylloides M.R.Almeida, Fl. Maharashtra 5A: 61. 2009, nom. superfl.

Flowering: August-December.

LIFE FORM: Terrestrial.

HaвiтAт: Found under bushes and rocky crevices in moist deciduous and dry deciduous forests between 500-600 m.

Distribution in Maharashtra: Chandrapur, Osmanabad and Yavatmal.

General Distribution: India (Andhra Pradesh, Odisha, Karnataka, Kerala, Maharashtra, Tamil Nadu, Jharkhand Chhattisgarh, Madhya Pradesh), Sri Lanka.

SPECIMENS EXAMINED: Chandrapur district, Amborthra, B.M.Wadhwa 137363 (BSI). Osmanabad district, Udgir, K.P.Janardhanan 100815 (BSI). Yavatmal district, Bhiwkund, S.Karthikeyan 156606 (BSI).

Habenaria stenopetala Lindl., Gen. Sp. Orchid. Pl. 319. 1835

Flowering: August-December.

LIFE FORM: Terrestrial.

НАвітAт: Found under the shade of semi-evergreen forests c. $600 \mathrm{~m}$.

Distribution in Maharashtra: Kolhapur and Satara.

General Distribution: India (Assam, Manipur, Mizoram, Nagaland, Arunachal Pradesh, Sikkim, West Bengal Himachal Pradesh, Jammu \& Kashmir, Uttarakhand, Andhra Pradesh, Odisha, Karnataka, Maharashtra, Bihar, Jharkhand), Nepal, China, Myanmar, Japan, Philippines, Thailand, Vietnam.

SPECIMENS EXAMINED: Kolhapur district, Radhanagari, A.R.Kulkarni s.n. (SUK). Satara district, Koyna WLS, Prajakta Pathare 203073 (BSI).

Habenaria suaveolens Dalzell in Hooker's J. Bot. Kew Gard. Misc. 2: 263. 1850. Fig. 7K.

Habenaria panchganiensis Santapau \& Kapadia in J. Bombay Nat. Hist. Soc. 54: 478. 1957.

FLOWERING: July-September.

LIFE FORM: Terrestrial.

HАBiтAT: It prefers to grow on exposed lateritic rocky plateaus.

Distribution in Maharashtra: Kolhapur, Satara and Sindhudurg.

LANKESTERIANA 18(1). 2018. (C) Universidad de Costa Rica, 2018. 
General Distribution: India (Goa, Karnataka, Maharashtra), endemic to Western Ghats.

SPECIMENS EXAMINED: Kolhapur district, Radhanagari, J.S.Jalal 200545 (BSI). Satara district, Mahabaleshwar, J.S.Jalal 200442 (BSI). Sindhudurg district, Chokul, J.S. Jalal 195004 (BSI).

Habenaria viridiflora (Sw.) R. Br. ex Spreng., Syst. Veg. (ed. 16) 3: 691. 1826. Orchis viridiflora Rottler ex Sw., Kongl. Vetensk. Acad. Nya Handl. 21: 206. 1800 .

Flowering: August-September.

LIFE FORM: Terrestrial.

HАВітAт: Reported from lateritic plateau of Malvan c. $50 \mathrm{~m}$.

Distribution in Maharashtra: Sindhudurg.

General Distribution: India (Assam, Karnataka, Kerala, Maharashtra, Tamil Nadu), Bangladesh, Sri Lanka, Myanmar, Thailand, Indo-China.

Note: This is reported by Stocks from Konkan and by Dalzell \& Gibson from Malvan. No subsequent workers have collected it again. There is no herbarium record available in $B L A T, B S I, S U K, C A L$ from Maharashtra.

\section{LIPARIS Rich.}

Liparis odorata (Willd.) Lindl., Gen. Sp. Orchid. P1.: 26. 1830. Fig. 7L.

Malaxis odorata Willd., Sp. P1. 4: 91. 1805.

Liparis dalzellii Hook.f., Fl. Brit. India 5: 698. 1890.

FLOWERING: June-September.

LIFE FORM: Terrestrial.

HaвiтAт: Found in semi-evergreen forests at elevations between 100-1200 m.

Distribution in Maharashtra: Kolhapur, Pune, Satara and Sindhudurg.

General Distribution: India (Assam, Manipur, Meghalaya, Mizoram, Nagaland, Arunachal Pradesh, Sikkim, West Bengal, Uttarakhand, Odisha, Andhra Pradesh, Goa, Karnataka, Kerala, Maharashtra, Tamil Nadu, Jharkhand), Bhutan, Nepal, China, Myanmar, Japan, Thailand, Laos, Vietnam, Pacific islands.

SPECIMENS EXAMINED: Kolhapur district, Radhanagari, J.S.Jalal 200523 (BSI). Pune district, Saltar dam, Lonavala, J.S.Jalal
200567 (BSI). Satara district, Mahabaleshwar, J.S.Jalal 200415 (BSI). Sindhudurg district, Sawantwadi, J.S.Jalal 195030 (BSI).

\section{LuISIA Gaudich.}

Luisia tenuifolia Blume in Rumphia 4: 50. 1849.

Luisia birchea Blume, Rumphia 4: 50. 1849.

Luisia evangelinae Blatt. \& McCann, J. Bombay Nat. Hist. Soc. 35: 493. 1932.

Luisia tenuifolia var. evangelinae (Blatt. \& McCann) Santapau \& Kapadia in J. Bombay Nat. Hist. Soc. 59: 829. 1962.

Luisia laurifolia M.R.Almeida, Fl. Maharashtra 5A: 66. 2009, nom. superfl.

Luisia laurifolia var. evangelinae (Blatt. \& McCann) M.R.Almeida, Fl. Maharashtra 5A: 66. 2009.

FLOWERING: March-April.

LIFE FORM: Epiphytic.

HaBitat: Found in semi-evergreen forests and moist deciduous forests between 100-600 m.

Distribution in Maharashtra: Satara and Thane.

General Distribution: India (Goa, Karnatak, Kerala, Maharashtra), Sri Lanka.

Specimens eXamined: Satara district, Koyna valley, Z.J.Kapadia 2912 (BLAT). Thane district, Tungar, N.Y.Das 3218 (BLAT).

Luisia trichorhiza (Hook.) Blume, Rumphia 4: 50. 1849.

Vanda trichorhiza Hook., Exot. Fl. 1: t. 72.1823.

FLOWERING: March-April.

LIFE FORM: Epiphyte.

HABiтAT: Found in dry deciduous forests.

Distribution in Maharashtra: Amravati and Gadchiroli.

General distribution: India (Assam, Meghalaya, Nagaland, Arunachal Pradesh, Sikkim, West Bengal, Uttarakhand, Andhra Pradesh, Odisha, Maharashtra, Tamil Nadu, Chhattisgarh, Jharkhand), Bangladesh, Bhutan, Nepal, Myanmar, Thailand.

Specimens examined: Amravati district, Amzari, G.D.Muratkar 2433 (BAMU). Gadchiroli district, Bhamaragad range, R.S.Govekar 197 (BAMU).

Luisia zeylanica Lindl., Fol. Orchid. 4: 3. 1853

Flowering: May-June. 


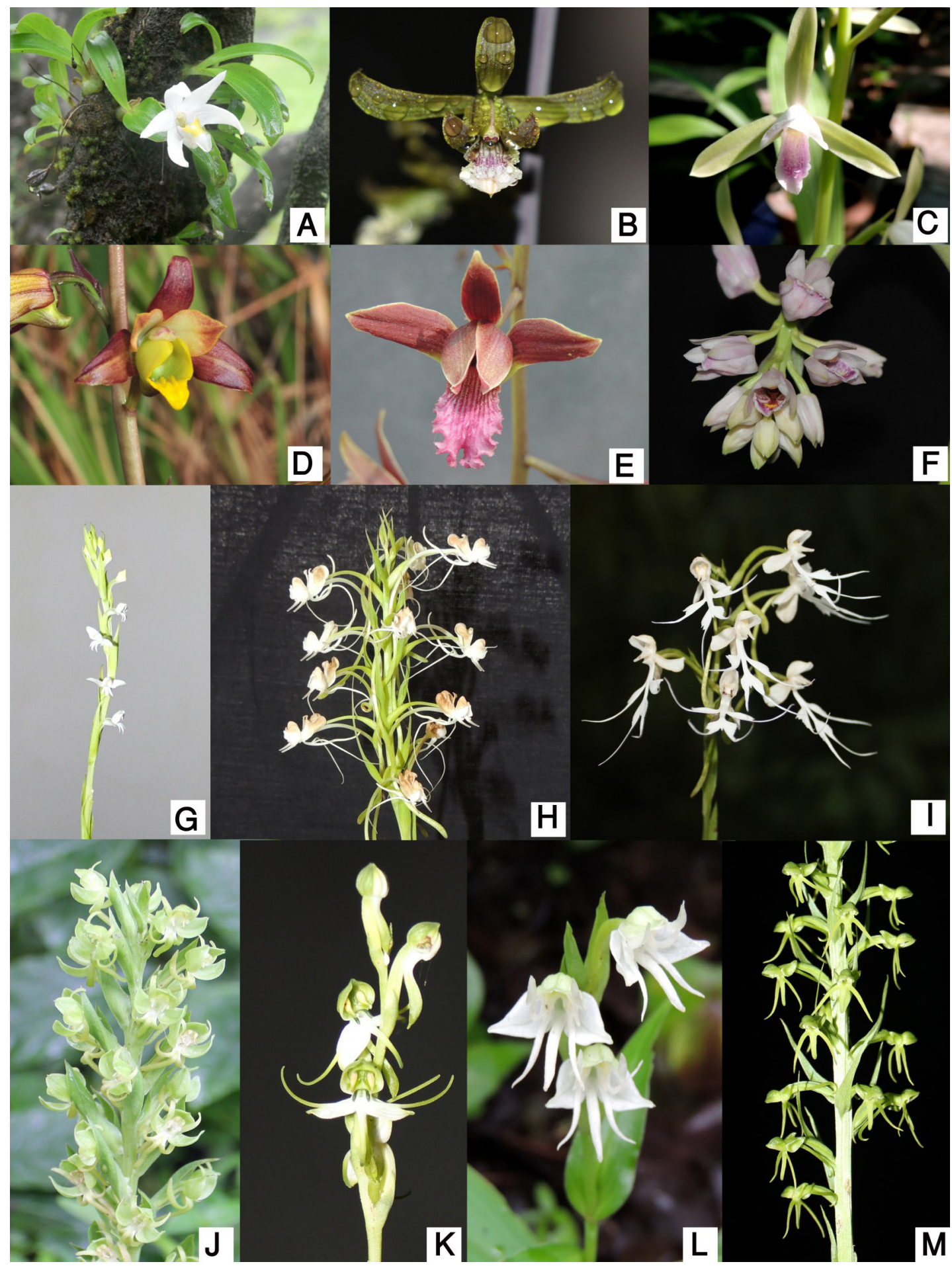

Figure 6. A. Conchidium reticosum, B. Eulophia graminea, C. Eulophia herbacea, D. Eulophia pratensis, E. Eulophia spectabilis, F. Geodorum densiflorum, G. Habenaria brachyphylla, H. Habenaria commelinifolia, I. Habenaria crinifera, J. Habenaria digitata, K. Habenaria diphylla, L. Habenaria foliosa, M. Habenaria furcifera. 


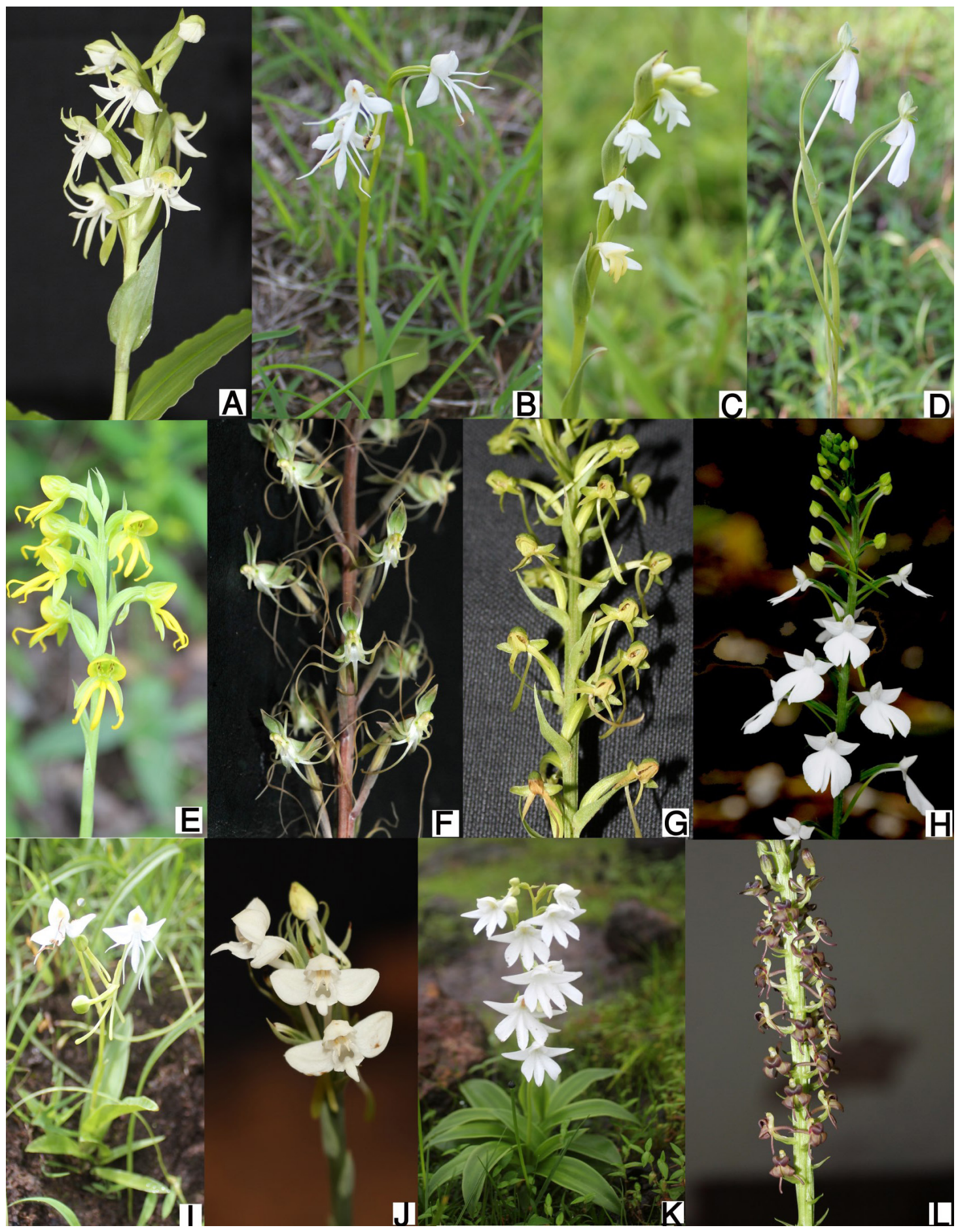

Figure 7. A. Habenaria gibsonii, B. Habenaria grandifloriformis, C. Habenaria heyneana, D. Habenaria longicorniculata, E. Habenaria marginata, F. Habenaria multicaudata, G. Habenaria ovalifolia, H. Habenaria plantaginea, I. Habenaria rariflora, J. Habenaria roxburghii, K. Habenaria suaveolens, L. Liparis odorata. 
LIFE FORM: Epiphyte.

НАвітAт: Found in dry deciduous forests and moist deciduous forests.

Distribution in Maharashtra: Amravati and Sindhudurg.

General distribution: India (Assam, Meghalaya, Manipur, Nagaland, Arunachal Pradesh, Goa, Karnataka, Maharashtra, Andaman \& Nicobar), Nepal, Myanmar, Indonesia, Malaysia, Thailand, Laos, Vietnam.

Specimens EXAmined: Amravati district, Chikhaldara, J.S.Jalal 200742 (BSI). Sindhudurg district, Sawantwadi, J.S.Jalal 194992 (BSI).

\section{Malaxis Sol. ex Sw.}

Malaxis versicolor (Lindl.) Abeyw., Ceylon J. Sci. Biol. Sci. 2: 247. 1959. Fig. 8A.

Microstylis versicolor Lindl., Gen. Sp. Orchid. P1. 21. 1830.

Corymborkis versicolor (Lindl.) M.R.Almeida, Fl. Maharashtra 5A: 30. 2009.

FLowering: July-August.

LIFE FORM: Terrestrial.

HaвiтAT: Found in shady localities of semievergreen forests and evergreen forests.

Distribution in Maharashtra: Kolhapur, Pune, Raigad, Ratnagiri, Satara and Sindhudurg.

General Distribution: India (Andhra Pradesh, Odisha, Maharashtra, Goa, Karnataka, Kerala, Tamil Nadu), Sri Lanka.

SPecimens eXAmined: Kolhapur district, Ajara, J.S.Jalal 200498 (BSI). Pune district, Ambavne, B.Venkata Reddi 99309 (BSI). Raigad district, Matheran, J.S.Jalal 200583 (BSI). Ratnagiri district, Shivapur, B.G.Kulkarni 129655 (BSI). Satara district, Mahabaleshwar, J.S.Jalal 200432 (BSI). Sindhudurg district, Amboli, J.S.Jalal 200488 (BSI).

Nervilia Comm. ex Gaudich.

Nervilia concolor (Blume) Schltr., Bot. Jahrb. Syst. 45: 404. 1911. Fig. 8B.

Cordyla concolor Blume, Bijdr. Fl. Ned. Ind.: 416. 1825. Nervilia aragoana Gaudich., Voy. Uranie 422, t.

35. 1829.
FLOWERING: May-August.

LIFE FORM: Terrestrial.

HABIтAT: Found in moist localities under the shade of deciduous forests, dry deciduous forests and semi-evergreen forests.

Distribution in Maharashtra: Kolhapur, Nandurbar, Nashik, Pune, Raigad, Ratnagiri, Sindhudurg and Thane.

General distribution: India (Assam, Meghalaya, Manipur, Nagaland, Sikkim, West Bengal, Himachal Pradesh, Uttarakhand, Andhra Pradesh, Odisha, Goa, Karnataka, Kerala, Tamil Nadu, Jharkhand, Uttar Pradesh, Rajasthan, Andaman \& Nicobar), Bangladesh, Bhutan, Nepal, China, Myanmar, Indonesia, Japan, Malaysia, New Guinea, Philippines, Thailand, Laos, Vietnam, Pacific islands, Australia.

SPECIMENS EXAMINED: Kolhapur district, Radhanagari, J.S.Jalal 200535 (BSI). Nandurbar district, Toranmal, 18.07.2014, J.S.Jalal 195134 (BSI). Nashik district, Near Saputara, J.S.Jalal 195141 (BSI). Pune district, Nane Ghat, J.S.Jalal 200838 (BSI). Raigad district, Supegaon, J.S.Jalal 200892 (BSI). Ratnagiri district, Dapoli, J.S.Jalal 194929 (BSI). Sindhudurg district, Sawantwadi, J.S.Jalal 200517 (BSI). Thane district, Tansa, J.S.Jalal 200858 (BSI).

Nervilia crociformis (Zoll. \& Moritzi) Seidenf., Dansk. Bot. Ark. 32: 151, f. 92.1978 . Fig. 8C.

Bulborchis crociformisZoll. \& Moritzi, Syst. Verz. 89. 1846.

Nervilia prainiana (King \& Pantl.) Seidenf., Dansk Bot. Ark. 32(2): 149. 1978.

FlOWERING: June-July.

LIFE FORM: Terrestrial.

НАвітAт: Found in moist deciduous forests, dry deciduous forests and semi-evergreen forests.

Distribution in Maharashtra: Kolhapur, Pune, Satara and Sindhudurg.

General distribution: India (Manipur, Arunachal Pradesh, Sikkim, Uttarakhand, Andhra Pradesh, Odisha, Goa, Karnataka, Kerala, Maharashtra, Tamil Nadu, Jharkhand), Nepal, China, Indonesia, Malaysia, Philippines, Thailand, Vietnam, New Guinea, Africa, Australia.

SPeCimens EXAmined: Kolhapur district, Chandgad, LANKESTERIANA 18(1). 2018. (C) Universidad de Costa Rica, 2018. 
J.S.Jalal 200974 (BSI). Pune district, Nane Ghat, J.S.Jalal 200839 (BSI). Satara district, Mahabaleshwar, J.S.Jalal 200429 (BSI). Sindhudurg district, Amboli, J.S.Jalal 200497 (BSI).

Nervilia infundibulifolia Blatt. \& McCann, J. Bombay Nat. Hist. Soc. 35: 725, t. 3. 1932. Fig. 8D.

Flowering: May-June.

LIFE FORM: Terrestrial.

HавітAт: Found in moist-shaded and damp places under dense shade.

Distribution in Maharashtra: Pune, Raigad, Thane and Sindhudurg.

General Distribution: India (Arunachal Pradesh, Uttarakhand, Odisha, Andhra Pradesh, Maharashtra, Karnataka, Kerala, Tamil Nadu Jharkhand), Myanmar, Indonesia, Malaysia, Thailand, Laos.

Specimens examined: Pune district, Amby valley, J.S.Jalal 200405 (BSI). Raigad district, Phansad, J.S.Jalal 200882 (BSI). Thane district, Malshej Ghat, J.S.Jalal 200846 (BSI). Sindhudurg district, Chaukul, J.S.Jalal 195003 (BSI).

Nervilia plicata (Andrews) Schltr., Bot. Jahrb. Syst. 45: 403. 1911. Fig. 8E.

Arethusa plicata Andrews, Bot. Repos. 5: 321. 1803.

Pogonia plicata (Andrews) Lindl., Gen. Sp. Orchid. P1.: 415. 1840.

Nervilia discolor (Blume) Schltr., Bot. Jahrb. Syst. 45: 403. 1911.

FLowering: May-August.

LIFE FORM: Terrestrial.

HaвiтAт: Found in moist deciduous forests, semievergreen forests.

Distribution in Maharashtra: Kolhapur, Pune, Sindhudurg and Thane.

General distribution: India (Assam, Manipur, Meghalaya, Mizoram, Arunachal Pradesh, Sikkim, West Bengal, Himachal Pradesh, Jammu \& Kashmir, Uttarakhand, Odisha, Gujarat, Karnataka, Kerala, Maharashtra, Tamil Nadu, Andaman \& Nicobar), Bangladesh, Bhutan, Nepal, China, Myanmar, Indonesia, Malaysia, Philippines, Thailand, Laos, Vietnam, New Guinea, Australia.
SPeCimens EXAmined: Kolhapur district, Tilari, Chandgad, J.S.Jalal 197711 (BSI). Pune district, Bhimashanker, Z.J.Kapadia 1464 (BLAT). Sindhudurg district, Amboli, J.S.Jalal 200489 (BSI). Thane district, Malshej Ghat, J.S.Jalal 200848 (BSI).

\section{Oberonia Lindl.}

Oberonia bicornis Lindl.,Gen. Sp. Orchid. Pl. 16. 1830.

FLowering: October-November.

LIFE FORM: Epiphyte.

HАВітAт: Found in semi-evergreen forests at higher plateaus.

Distribution in Maharashtra: Sindhudurg.

General Distribution: India (Mizoram, Meghalaya, Karnataka, Kerala, Maharashtra, Tamil Nadu), Bangladesh.

Specimen eXamined: Sindhudurg district, Chaukul, B.G.Gavade 61 (BLAT).

Oberonia brunoniana Wight, Icon. P1. Ind. Orient. 5

(1): t. 1662. 1851. Fig. 8F.

FLOWERING: July-October.

LIFE FORM: Epiphyte.

HABiтAT: Found under the shade of semi-evergreen forests.

Distribution in Maharashtra: Kolhapur and Thane.

General Distribution: India (Andhra Pradesh, Goa, Karnataka, Kerala, Maharashtra, Tamil Nadu, Dadara \& Nagar Haveli), endemic to Western Ghats.

SPecimens EXAMIned: Satara district, Koyna, J.S.Jalal 200772 (BSI). Thane district, Kasa, Yadav \& Soman 2600 (Sardar Patel University Herbarium).

Oberonia ensiformis (Sm.) Lindl., Fol. Orchid. 8: 4. 1859.

Malaxis ensiformis Sm. in A.Rees, Cycl. 22: n. ${ }^{\circ} 14$. 1812.

FLOWERING: December-January.

LIFE FORM: Epiphyte.

HАВiтAT: Found under the shade of moist deciduous forests.

Distribution in Maharashtra: Thane.

General distribution: India (Manipur, Meghalaya, Mizoram, Nagaland, Arunachal Pradesh, Sikkim, 
West Bengal, Uttarakhand, Andhra Pradesh, Odisha, Karnataka, Kerala, Maharashtra, Tamil Nadu, Andaman \& Nicobar), Nepal, China, Myanmar, Thailand, Laos, Vietnam.

SPecimen EXAmined: Thane district, Usgaon, N.Y.Das 3203 (BLAT).

Oberonia falconeri Hook. f. in Hooker's Icon. Pl. Ind. Orient. Pl. 18: t. 1780. 1888.

Flowering: August-September.

LIFE FORM: Epiphyte.

НАвітAт: Found in dry deciduous forests and moist deciduous forests.

Distribution in Maharashtra: Thane.

General Distribution: India (Assam, Meghalaya, Arunachal Pradesh, Sikkim, West Bengal, Uttarakhand, Odisha, Andhra Pradesh, Karnataka, Maharashtra, Bihar, Jharkhand, Chhattisgarh, Madhya Pradesh), Bangladesh, Nepal, China, Malaysia, Thailand, Laos, Vietnam.

Specimen eXamined: Thane district, Tansa, Z.J.Kapadia 731 (BLAT).

Oberonia mucronata (D.Don) Ormerod \& Seidenf. in

G.Seidenfaden, Contr. Orchid Fl. Thailand 13: 20. 1997.

Stelis mucronata D.Don, Prodr. Fl. Nepal.: 32. 1825.

Oberonia denticulata Wight, Icon. Pl. Ind. Orient. 5: t. 1625. 1851.

Oberonia iridifolia var. denticulata (Wight) Hook.f., Fl. Brit. India 5: 676. 1890.

FLOWERING: September-October.

LIFE FORM: Epiphyte.

HABITAT: Found in moist deciduous forests.

Distribution in Maharashtra: Thane.

General distribution: India (Assam, Manipur, Meghalaya, Mizoram, Nagaland, Arunachal Pradesh, Sikkim, West Bengal, Andhra Pradesh, Odisha, Gujarat, Goa, Kerala, Tamil Nadu, Maharashtra, Chhattisgarh, Andaman \& Nicobar), Bangladesh, Bhutan, Nepal, China, Myanmar, Indonesia, Malaysia, Philippines, Laos.

Specimen eXamined: Thane district, Tansa, Z.J.Kapadia 1638 (BLAT).

Oberonia recurva Lindl., Edwards's Bot. Reg. 25(Misc.): 14. 1839. Fig. 8G.
Oberonia recurva var. lingmalensis (Blatt. \& McCann) Santapau \& Kapadia, J. Bombay Nat. Hist. Soc. 57: 259. 1960.

FLOWERING: October-March.

LIFE FORM: Epiphyte.

НАвітAт: Found in dry deciduous forests, moist deciduous forests and semi-evergreen forests.

Distribution in Maharashtra: Kolhapur, Pune, Ratnagiri, Satara and Sindhudurg.

GenERAL Distribution: India (Meghalaya, Sikkim, West Bengal, Karnataka, Kerala, Maharashtra), Bhutan, Nepal, Sri Lanka, China, Thailand.

Specimens eXamined: Kolhapur district, Chandgad, J.S.Jalal 194986 (BSI). Pune district, Bhimashankar, J.S.Jalal 200725 (BSI). Ratnagiri district, Mandangad, J.S.Jalal 200733 (BSI). Satara district, Mahabaleshwar, J.S.Jalal 200412 (BSI). Sindhudurg district, Khamdadev plateau, J.S.Jalal 195015 (BSI).

Oberonia verticillata Wight, Icon. Pl. Ind. Orient. 5:

t. 1626.1851.

FLOWERING: September-October.

LIFE FORM: Epiphyte.

HавітAт: Found in dry deciduous forest on Ficus tree.

Distribution in Maharashtra: Thana.

General Distribution: India (Goa, Karnataka, Kerala, Maharashtra, Tamil Nadu), endemic to Western Ghats.

Specimen examined: Thana: Kasa, K.V.S. Badarinath 4021 (Sardar Patel University Herbarium, Gujarat).

\section{Pachystoma Blume}

Pachystoma pubescens Blume, Bijdr. 376, t. 3, f. 3. 1825.

FLOWERING: February-March.

LIFE FORM: Terrestrial.

Habitat: Found in semi-evergreen forests in open situation.

Distribution in Maharashtra: Satara.

General distribution: India (Manipur, Meghalaya, Mizoram, Nagaland, Arunachal Pradesh, Sikkim, West Bengal, Himachal Pradesh, Uttarakhand, Odisha, Karnataka, Kerala, Maharashtra, Tamil Nadu, Madhya Pradesh, Punjab, Uttar Pradesh, Andaman \& Nicobar), LANKESTERIANA 18(1). 2018. (C) Universidad de Costa Rica, 2018. 
Bangladesh, Bhutan, Nepal, Myanmar, Indonesia, Malaysia, Philippines, Indo-China, New Guinea, Australia.

Specimen examined: Satara district, Koyna, Bachulkar 323 (SUK).

\section{Pecteilis Raf.}

Pecteilis gigantea (Sm.) Rafin., Fl. Tell. 2: 38. 1837. Fig. $8 \mathrm{H}$.

Orchis gigantea Sm., Exot. Bot. 2: 79. 1806.

FLowering: September-October.

LIFE FORM: Terrestrial.

HABITAT: Found in open grassy slopes and plateaus of moist deciduous forests, semi-evergreen forests along margins of forests in open and usually on slightly sloping grounds.

Distribution IN MAharashtra: Bhandara, Kolhapur, Nashik, Pune, Raigad, Satara, Sindhudurg and Thane.

General Distribution: India (Nagaland, Himachal Pradesh, Jammu \& Kashmir, Uttarakhand, Andhra Pradesh, Odisha, Goa, Gujarat, Karnataka, Kerala, Maharashtra, Tamil Nadu, Dadara \& Nagar Haveli, Madhya Pradesh, Chhattisgarh, Bihar, Jharkhand), Pakistan, Myanmar.

SPecimens eXAmined: Bhandara district, Kusumtondi, S.K.Malhotra 144689 (BSI). Kolhapur district, Radhanagari, J.S.Jalal 200526 (BSI). Nashik district, Kumbhala, John Cherian 112620 (BSI). Pune district, Mulshi, J.S.Jalal 195078 (BSI). Raigad district, Matheran, J.S.Jalal 200703 (BSI). Satara district, Mahabaleshwar, J.S.Jalal 200420 (BSI). Sindhudurg district, Kudal, J.S.Jalal 200513 (BSI). Thane district, Tansa, J.S.Jalal 200855 (BSI).

\section{Peristylus Blume}

Peristylus aristatus Lindl.,Gen. Sp. Orchid. P1.: 300. 1835. Fig. 8I.

FLOWERING: July-September.

LIFE FORM: Terrestrial.

НАвітAт: Found under the shade of semi-evergreen forests.

Distribution in Maharashtra: Kolhapur, Satara and Sindhudurg.
General Distribution: India (Goa, Karnataka, Kerala, Maharashtra, Tamil Nadu), Nepal, Sri Lanka, Myanmar.

Specimens examined: Kolhapur district, Gajapur, M.M.Sardesai 201 (SUK). Satara district, Shirshingi, R.K.Kochhar 153668 (BSI). Sindhudurg district, Amboli, J.S.Jalal 200479 (BSI).

Peristylus constrictus (Lindl.) Lindl., Gen. Sp. Orchid. P1.: 300. 1835.

Herminium constrictum Lindl., Edwards's Bot. Reg. 18: t. 1499. 1832.

FLOWERING: August-September.

LIFE FORM: Terrestrial.

Habitat: Found in grassy slopes of dry deciduous forests.

Distribution in Maharashtra: Amravati.

General distribution: India (Assam, Meghalaya, Nagaland, Arunachal Pradesh, Sikkim, West Bengal, Himachal Pradesh, Jammu \& Kashmir, Uttarakhand, Odisha, Maharashtra, Bihar, Jharkhand, Madhya Pradesh, Uttar Pradesh), Bangladesh, Bhutan, Nepal, China, Myanmar, Philippines, Thailand, Cambodia, Vietnam.

Specimen eXAmined: Amravati district, Semadoh, M.Y.Ansari 149372 (BSI).

Peristylus densus (Lindl.) Santapau \& Kapadia in J. Bombay Nat. Hist. Soc. 57: 128. 1960. Fig. 8J.

Coeloglossum densum Lindl., Gen. Sp. Orchid. P1.: 302. 1835.

Flowering: August-September.

LIFE FORM: Terrestrial.

НАвітAт: Found in high altitude rocky plateaus.

Distribution in Maharashtra: Kolhapur, Pune, Satara, Sindhudurg and Thane.

GeNerAL Distribution: India (Meghalaya, Nagaland, Arunachal Pradesh, Sikkim, Goa, Karnataka, Kerala, Maharashtra, Tamil Nadu), Bangladesh, Nepal, Sri Lanka, China, Myanmar, Thailand, Cambodia, Vietnam.

SPECimENS EXAmined: Kolhapur district, Chandgad, J.S.Jalal 194977 (BSI). Pune district, Mulshi, B.Venkata Reddi 99065 (BSI). Satara district, Mahabaleshwar, M.Y.Ansari 67726 (BSI). Sindhudurg district, Jalvadi-Chokul, J.S.Jalal 195006 (BSI). Thane district, Tokavada range, K.V.Billore 115809 (BSI). 
Peristylus lawii Wight, Icon. P1. Ind. Orient. 5: t. 1695. 1851.

Flowering: July-September.

Habit AND Habitat: Terrestrials, found under the shade of moist deciduous forests and dry deciduous forests between 200-500 m.

Distribution in Maharashtra: Thane and Yavatmal.

General Distribution: India (Uttarakhand, Odisha, Gujarat, Karnataka, Kerala, Maharashtra, Tamil Nadu, Bihar, Jharkhand, Madhya Pradesh), Nepal, Myanmar.

SPECIMENS EXAMINED: Thane district, Tansa, J.S.Jalal 200861 (BSI). Yavatmal district, Patan Bori Range, S. Karthikeyan 160088 (BSI).

Peristylus plantagineus (Lindl.) Lindl., Gen. Sp. Orchid. P1.: 300. 1835.

Herminium plantagineum Lindl., Edwards's Bot. Reg. 18: t. 1499. 1832.

FLOWERING: August-October.

LIFE FORM: Terrestrial.

Habitat: Found in dry deciduous forests, moist deciduous forests, semi-evergreen forests.

Distribution IN MAHARAshtRa: Chandrapur, Gadchiroli, Kolhapur, Pune, Raigad, Satara, and Sindhudurg.

General Distribution: India (West Bengal, Odisha, Andhra Pradesh, Goa, Gujarat, Karnataka, Kerala, Maharashtra, Tamil Nadu, Chhattisgarh, Madhya Pradesh), Sri Lanka.

Specimens examined: Chandrapur district, Amborthra, B.M.Wadhwa 137363 A (BSI). Gadchiroli district, R.S.Govekar 1284 (BAMU). Kolhapur district, Chandgad, J.S.Jalal 194984 (BSI). Pune district, Nane Ghat, J.S.Jalal 200841 (BSI). Raigad district, Matheran, J.S.Jalal 200701 (BSI). Sindhudurg district, Amboli, J.S.Jalal 200482 (BSI).

Peristylus stocksii (Hook.f.) Kraenzl., Orchid. Gen. Sp. 1: 513. 1898. Fig. 8K.

Habenaria stocksii Hook.f., Fl. Brit. India 6: 158. 1890.

FLOWERING: July-September.

LIFE FORM: Terrestrial.

НАвітAт: Found in dry deciduous forests, moist deciduous forests and semi-evergreen forests.
Distribution IN MAHARAShtra: Amravati, Kolhapur, Nashik, Pune, Raigad, Ratnagiri and Thane.

General Distribution: India (Goa, Gujarat, Karnataka, Maharashtra, Tamil Nadu, Bihar, Madhya Pradesh), endemic to India.

Specimens examined: Amravati district, Melghat, M.Y.Ansari 149371 (BSI). Kolhapur district, Chandgad, J.S.Jalal 194983 (BSI). Nashik district, Kasara ghat, J.S.Jalal 200873 (BSI). Pune district, Rajmachi, J.S.Jalal 200577 (BSI). Raigad district, Matheran, J.S.Jalal 200597 (BSI). Ratnagiri district, Phonda ghat, R.S.Rao 129680 (BSI). Thane district, Malshej ghat, J.S.Jalal 200844 (BSI).

\section{РноLidota Lindl.}

Pholidota imbricata Lindl. in W.J.Hooker, Exot. Fl. 2: t. 138.1825.

FLOWERING: July-September.

LIFE FORM: Epiphyte.

HАВітAT: Found in semi-evergreen forests.

Distribution in MaHarashtra: Gadchiroli, Kolhapur and Sindhudurg.

General Distribution: India (Assam, Manipur, Meghalaya, Mizoram, Nagaland, Tripura, Arunachal Pradesh, Sikkim, West Bengal, Uttarakhand, Andhra Pradesh, Odisha, Goa, Karnataka, Kerala, Maharashtra, Tamil Nadu, Jharkhand, Chhattisgarh, Madhya Pradesh, Andaman \& Nicobar), Bangladesh, Bhutan, Nepal, Pakistan, Sri Lanka, China, Myanmar, Indonesia, Malaysia, Thailand, New Guinea, Pacific islands, Australia.

Specimens eXamined: Gadchiroli district, Binagunda, R.S.Govekar 1703 (BAMU). Kolhapur district, Patgaon, M.M.Sardesai 2043 (SUK). Sindhudurg district, Virdi, Sawantwadi, S.M.Almeida 4895 (BLAT).

Pinalia Lindl.

Pinalia mysorensis (Lindl.) Kuntze, Revis. Gen. Pl. 2: 679. 1891.

Eria mysorensis Lindl., J. Proc. Linn. Soc., Bot. 3: 54. 1858.

FLOWERING: July-October. 
LIFE FORM: Epiphyte.

НАвітAт: Found in semi-evergreen forests.

Distribution in Maharashtra: Satara.

General Distribution: India (Karnataka, Kerala, Maharashtra, Tamil Nadu), endemic to Western Ghats.

Specimen eXamined: Satara district, Mahabaleshwar, 16.08.1883, J.M.Woodrow s.n. (CAL acc.n. 453115).

Note: This species was collected by J.M.Woodrow from Mahabaleshwar in 1883. After that it was not collected by any subsequent workers from Mahabaleshwar or any other area from Maharashtra.

Pinalia polystachya (A.Rich.) Kuntze, Revis. Gen. Pl. 2: 679. 1891.

Eria polystachya A.Rich., Ann. Sci. Nat., Bot., sér. 2, 15: 20. 1841.

FLowering: July-October.

LIFE FORM: Epiphyte.

НАВітAт: Found in semi-evergreen forests.

Distribution in Maharashtra: Satara.

General Distribution: India (Karnataka, Kerala, Maharashtra, Tamil Nadu), endemic to Western Ghats.

Specimen eXAmined: Satara district, Koyna valley, July 1892. Herb. Cooke no. 447 (BSI).

Note: This species is endemic to Western Ghats. It was reported from Koyna valley in 1892. Only single specimen of this taxon is available in herbarium $B S I$.

Plectoglossa (Hook.f.) K. Prasad \&Venu

Plectoglossa perrottetiana (A. Rich.) K. Prasad \& Venu, Rheedea 88. 2015.

Habenaria perrottetiana A. Rich. in Ann. Sci. Nat., Bot., 2,15:74, t. 4 B. 1841.

Flowering: August-October.

Habitat And ECOlogy: Terrestrials, found in open grassy slopes of higher plateaus.

Distribution in Maharashtra: Kolhapur.

General distribution: India (Karnataka, Kerala, Maharashtra, Tamil Nadu), endemic to Western Ghats.

Specimen examined: Kolhapur district, Amba, M.M.Sardesai 4341 (SUK).
Porpax Lindl.

Porpax jerdoniana (Wight) Rolfe, Orchid Rev. 16: 8. 1908.

Lichenora jerdoniana Wight, Icon. Pl. Ind. Orient. 5: t. 1738. 1851.

FLOWERING: June-July.

LIFE FORM: Epiphyte.

Haвitat: Found in moist deciduous and semievergreen forests.

Distribution in Maharashtra: Kolhapur, Thane, Ratnagiri and Sindhdurg.

General Distribution: India (Goa, Maharashtra, Karnataka, Kerala, Tamil Nadu, Andaman \& Nicobar), endemic to India.

SPECimENS EXAMINED: Kolhapur district, Tilari, J.S.Jalal 197746 (BSI). Ratnagiri district, Kudavale, J.S.Jalal 194921 (BSI). Thane district, Usgaon, N.Y.Das 1704 (BLAT). Sindhudurg district, Amboli, M.R.Almeida 334 (BLAT).

Porpax reticulata Lindl., Edwards's Bot. Reg. 31(Misc.): 62. 1845. Fig. 8L.

FLOWERING: April-June.

LIFE FORM: Epiphyte.

Haвitat: Found in moist deciduous forests and semi-evergreen forests.

Distribution in Maharashtra: Kolhapur, Pune, Raigad, Ratnagiri and Sindhudurg.

General Distribution: India (Goa, Maharashtra, Karnataka, Kerala, Tamil Nadu), Thailand, Laos, Vietnam.

Specimens eXamined: Kolhapur district, Chandgad, J.S.Jalal 194970 (BSI). Pune district, Khandala, H.Santapau 743 (BLAT). Raigad district, Matheran, N.A.Irani 4314 (BLAT). Ratnagiri district, Kudavale, J.S.Jalal 194922 (BSI). Sindhudurg district, Amboli, J.S.Jalal 200477 (BSI).

\section{Rhynchostylis Blume}

Rhynchostylis retusa (L.) Blume, Bijdr. 286. 1825.

Epidendrum retusum L., Sp. Pl.: 953. 1753.

FLOWERING: June-July.

LIFE FORM: Epiphyte.

HaвiтAт: Found in dry deciduous forests and moist deciduous forests. 
Distribution in Maharashtra: Amravati, Gadchiroli, Kolhapur, Nashik, Mumbai Suburban, Pune, Raigad, Ratnagiri and Thane.

General distribution: India (Assam, Manipur, Meghalaya, Mizoram, Nagaland, Tripura, Arunachal Pradesh, Sikkim, West Bengal, Himachal Pradesh, Jammu \& Kashmir, Uttarakhand, Andhra Pradesh, Odisha, Goa, Gujarat, Karnataka, Kerala, Maharashtra, Tamil Nadu, Jharkhand, Chhattisgarh, Haryana, Madhya Pradesh, Andaman \& Nicobar), Bangladesh, Bhutan, Nepal, Sri Lanka, China, Myanmar, Indonesia, Malaysia, Philippines, Thailand, Indo-China.

SPeCimens EXAmined: Amravati district, Semadoah, J.S.Jalal 200753 (BSI). Gadchiroli district, R.S.Govekar s.n. (BAMU). Kolhapur district, Patgaon, M.M.Sardesai 1310 (SUK). Mumbai Suburban district, Malad, G.L.Shah 4626 (BLAT). Nashik district, Umberthan, P.L.Narasimhan 165454 (BSI). Pune district, Khandala, J.S.Jalal 195041 (BSI). Raigad district, Karjat, J.S.Jalal 195049 (BSI). Ratnagiri district, Dapoli, J.S.Jalal 194937 (BSI). Thane district, Tansa, J.S.Jalal 200864 (BSI).

\section{Smithsonia C.J.Saldanha}

Smithsonia maculata (Dalzell) C.J.Saldanha, J. Bombay Nat. Hist. Soc. 71: 74. 1974.

Micropera maculata Dalzell, Hooker's J. Bot. Kew Gard. Misc. 3: 282. 1851.

Flowering: May-June.

LIFE FORM: Epiphyte.

Distribution in Maharashtra: Sindhudurg.

General Distribution: India (Maharashtra, Karnataka, Kerala and Tamil Nadu), endemic to Western Ghats.

NotE: T. Cooke has reported this species from 'Phondaghat' (in Kankavli taluka) based on the collection by Ritchie. There is no herbarium record in BSI, CAL and BLAT of this species. We have not seen any wild population of this plant in Maharashtra.

Smithsonia straminea C.J.Saldanha, J. Bombay Nat.

Hist. Soc. 71: 73. 1974.

FLowering: May-June.
LIFE FORM: Epiphyte.

НАВітAт: Found in semi-evergreen forests.

Distribution in Maharashtra: Ratnagiri.

General distribution: India (Goa, Karnataka, Kerala, Maharashtra), endemic to Western Ghats.

SPECIMENS EXAMINED: Ratnagiri district, Vigavani, J.S.Jalal 194912 (BSI).

Smithsonia viridiflora (Dalzell) C.J.Saldanha, J. Bombay Nat. Hist. Soc. 71: 75. 1974. Fig. 8M.

Micropera viridiflora Dalzell, Hooker's J. Bot. Kew Gard. Misc. 3: 282. 1851.

Aerides dalzelliana (Santapau) Garay, Bot. Mus. Leafl. 23: 158. 1972.

Flowering: May-June.

LIFE FORM: Epiphyte.

HАвітAт: Found in semi-evergreen and evergreen forests.

Distribution in Maharashtra: Kolhapur, Pune, Satara and Sindhudurg.

General Distribution: India (Goa, Karnataka, Kerala, Maharashtra), endemic to Western Ghats.

Specimens examined: Kolhapur district, Amba, R.K.Bhide s.n. $(B S I)$. Pune district, Dudhivare khind, J.S.Jalal 195071 (BSI). Satara district, Koyna, J.S.Jalal 200796 (BSI). Sindhudurg district, Amboli, J.S.Jalal 200403 (BSI).

SPIRANThes Rich.

Spiranthes sinensis (Pers.) Ames, Orchidaceae 2: 53. 1908.

Neottia sinensis Pers., Syn. Pl. 2: 511. 1807.

Spiranthes lancea (Thunb. ex Sw.) Bakh.f. \& Steenis, Blumea 6: 361. 1950.

FLOWERING: March - September.

LIFE FORM: Terrestrial.

Distribution in Maharashtra: Satara.

General Distribution: India (Assam, Manipur, Meghalaya, Mizoram, Nagaland, Tripura, Arunachal Pradesh, Sikkim, West Bengal, Himachal Pradesh, Jammu \& Kashmir, Uttarakhand, Odisha, Punjab, Karnataka, Kerala), Afghanistan, Bangladesh, Bhutan, Nepal, China, Myanmar, Japan, Kashmir, Korea, Malaysia, Mongolia, Philippines, Thailand, Vietnam, Pacific islands, Russia, Australia.

LANKESTERIANA 18(1). 2018. (C) Universidad de Costa Rica, 2018. 
Note: This species is included based on Santapau and Kapadia (1966). They discovered it by an accident. Mrs. Rukminibai collected a group of lily bulbs from Panchgani and planted in St. Xavier's College terrace garden. In the subsequent year during the month of March it was flowering along with lilies plants. The specimen number 1916 by Kapadia (BLAT) couldn't be traced in BLAT Harbarium. It was also reported by Stocks from Konkan region.

\section{Thunia Rchb.f.}

Thunia alba (Lindl.) Rchb.f. var. bracteata (Roxb.) N.Pearce \& P.J.Cribb in Edinb. J. Bot. 58: 116. 2001. Limodorum bracteatum Roxb., Fl. Ind., ed. 1832, 3: 466. 1832.

Thunia venosa Rolfe, Orchid Rev. 12: 206. 1905.

Flowering: August-September.

LIFE FORM: Epiphyte.

НавітAт: Found in semi evergreen forests.

Distribution in Maharashtra: Kolhapur, Pune and Satara.

Genaral distribution: India (Meghalaya, Arunachal Pradesh, Sikkim, West Bengal, Himachal Pradesh, Uttarakhand, Andhra Pradesh, Odisha, Kerala, Maharashtra), Bhutan, Nepal.

Specimens eXamined: Kolhapur district, Chandgad, J.S.Jalal 194971 (BSI). Pune district, Ambavne, B.Venkata Reddi 99020 (BSI). Satara district, Mahabaleshwar, M.M.Sardesai 1422 (SUK).

VANDA Jones ex R. Br.

Vanda tessellata (Roxb.) Hook. ex G.Don in J.C.Loudon, Hort. Brit.: 372. 1830. Fig. 8N.

Epidendrum tessellatum Roxb., P1. Coromandel 1: 34. 1795.

FLOWERING: March-June.

LIFE FORM: Epiphyte.

НАвітат: Epiphytes, found in moist deciduous forests and dry deciduous forests.

Distribution in Maharashtra: Amravati, Bhandara, Chandrapur, Gadchiroli, Nandurbar and Yavatmal.

General Distribution: India (Assam, Nagaland, Tripura, Arunachal Pradesh, Sikkim, West
Bengal, Uttarakhand, Andhra Pradesh, Odisha, Goa, Gujarat, Karnataka, Kerala, Maharashtra, Tamil Nadu, Dadara \& Nagar Haveli, Bihar, Jharkhand, Madhya Pradesh, Chhattisgarh, Uttar Pradesh, Rajasthan), Bangladesh, Nepal, Sri Lanka, Myanmar.

Specimens examined: Amravati district, Tarubanda, J.S.Jalal 200771 (BSI). Bhandara district, Nagzira, S.K.Malhotra 145137 (BSI). Chandrapur district, Bhawragarh, S.R.Rolla 96586 (BSI). Gadchiroli district, R.S.Govekar 0001 (BAMU). Nandurbar district, Toranmal, J.S.Jalal 195117 (BSI). Yavatmal district, Kharbi range, $S$. Karthikeyan 156720 (BSI).

Vanda testacea (Lindl.) Rchb. f., Gard Chron.2: 166.1877.

Aerides testacea Lindl., Gen. Sp. Orchid. P1.: 238. 1833.

Aerides wightiana Lindl., Gen. Sp. Orchid. Pl.: 238. 1833.

Vanda testacea var. parviflora (Lindl.)

M.R.Almeida, Fl. Maharashtra 5A: 90. 2009.

FLOWERING: May-June.

LIFE FORM: Epiphyte.

HАвітAт: Found in moist deciduous forests and dry deciduous forests.

Distribution In Maharashtra: Amravati, Gadchiroli, Thane, Nandurbar, Nashik, Raigad and Ratnagiri.

General Distribution: India (Assam, Manipur, Mizoram, Nagaland, Arunachal Pradesh, Sikkim, West Bengal, Himachal Pradesh, Uttarakhand, Andhra Pradesh, Odisha, Goa, Karnataka, Kerala, Maharashtra, Tamil Nadu, Jharkhand, Madhya Pradesh, Chhattisgarh, Uttar Pradesh, Rajasthan), Nepal, Sri Lanka, Myanmar.

Specimens eXAmined: Amravati district, Melghat, J.S.Jalal 200747 (BSI). Gadchiroli district, R.S.Govekar 1691 (BAMU). Thane district, Tansa, J.S.Jalal 200864 (BSI). Nandurbar district, Toranmal, J.S.Jalal 195120 (BSI). Nashik district, Pimpalsonda, P.L.Narasimhan 166395 (BSI). Raigad district, Talerwarphata, J.S.Jalal 194902 (BSI). Ratnagiri district, Dapoli, J.S.Jalal 194934 (BSI). 




Figure 8. A. Malaxis versicolor, B. Nervilia concolor, C. Nervilia crociformis, D. Nervilia infundibulifolia, E. Nervilia plicata, F. Oberonia brunoniana, G. Oberonia recurva, H. Pecteilis gigantea, I. Peristylus aristatus, J. Peristylus densus, K. Peristylus stocksii, L. Porpax reticulata, M. Smithsonia viridiflora, N. Vanda tessellata. 


\section{ZeuXine Lindl.}

Zeuxine gracilis (Breda) Blume, Coll. Orchid.: 69. 1858. Psychechilos gracilis Breda, Gen. Sp. Orchid. Asclep. 2: t. 9. 1829.

FLOWERING: February-March.

LIFE FORM: Terrestrial.

HАBiтAт: Found in semi-evergreen forests.

Distribution in Maharashtra: Kolhapur.

General Distribution: India (Meghalaya, Nagaland, Arunachal Pradesh, Odisha, Karnataka, Kerala, Maharashtra, Tamil Nadu), Indonesia, Malaysia, Thailand, Vietnam.

Specimen eXamined: Kolhapur district, Dajipur, M.M.Sardesai 2045 (SUK).

Zeuxine longilabris (Lindl.) Trimen, Syst. Cat. Fl. P1. Ceylon: 90. 1885.

Monochilus longilabris Lindl., Gen. Sp. Orchid. P1.: 487. 1840.

FLOWERING: February.

LIFE FORM: Terrestrial.

HАвітAт: Found in semi-evergreen forests and evergreen forests.

Distribution in Maharashtra: Kolhapur and Sindhudurg.

General Distribution: India (Assam, Tripura, Arunachal Pradesh, West Bengal, Odisha, Goa, Karnataka, Kerala, Maharashtra, Tamil Nadu, Bihar), Bangladesh, Nepal, Sri Lanka, Myanmar, Malaysia, Thailand, Cambodia.

Specimens eXamined: Kolhapur district, Pargad road, J.S.Jalal 195197 (BSI). Sindhudurg district, Amboli, J.S.Jalal 194948 (BSI).

Zeuxine strateumatica (L.) Schltr., Bot. Jahrb. 45 394. 1911.

Orchis strateumatica L. Sp. Pl. 2: 943. 1753.

FLOWERING: January-March.

LIFE FORM: Terrestrial.

Habitat: Found in marshy and sandy localities especially along river banks among the grasses.

Distribution in MAhARASHTRA: Ahmednagar, Akola, Kolhapur, Pune and Solapur.

General Distribution: India (Assam, Manipur, Meghalaya, Mizoram, Nagaland, Tripura, Arunachal Pradesh, Sikkim, West Bengal, Himachal Pradesh, Uttarakhand, Odisha, Andhra Pradesh, Gujarat, Karnataka, Kerala,
Maharashtra, Tamil Nadu, Bihar, Jharkhand, Delhi, Madhya Pradesh, Punjab, Rajasthan, Uttar Pradesh, Andaman \& Nicobar), Afghanistan, Bangladesh, Bhutan, Nepal, Pakistan, Sri lanka, China, Myanmar, Indonesia, Japan, Philippines, Malaysia, Thailand, Indo-China, West Asia, New Guinea, Africa, America.

SPECIMENS EXAMINED: Ahmednagar district, Khandgaon, R.Shinde 735 (BLAT). Akola district, Patur, S.Y.Kamble 152782 (BSI). Kolhapur district, Shivaji University campus, J.S.Jalal 200732 (BSI). Pune district, Mulshi, R.V.Kammathy 78359 (BSI). Solapur district, Ujani, S.K. Das Das 179635 (BSI).

\section{IMPERFECTLY KNOWN SPECIES}

Habenaria caranjensis Dalzell has uncertain identity and status. There is no specimen in any herbaria and it was not collected by any other botanist after Dalzell (Dalzell \& Gibson 1861). Santapau and Kapadia (1966) stated that the original locality given by Dalzell is the islands of 'Carunja near Bombay'. Carunja is a place near the Bombay coast and not an island. Moreover, at present the area belongs to Indian Navy and converted for Navy operations with port and township. Hence, existence of this species is doubtful.

\section{Excluded Taxa}

These species have been reported from Maharashtra by previous workers but are not included in our Checklist for the reasons given.

Acampe rigida (Buch.-Ham. ex Sm.) P.F.Hunt was reported by Singh \& Dawre (1983) based on collection numbers R.D.Pataekar 102001 (BSI) and H.P.Paranjape s.n. (BSI), which has been re-identified as Acampe praemorsa (Roxb.) Blatt. \& McCann.

Diplocentrum congestum Wight was included by Barbhuiya and Salunkhe (2016) in orchids of Maharashtra based on collection number S.M.Almeida 601 (BLAT), which was reidentified as Smithsonia straminea C.J.Saldanha.

Liparis dalzellii Hook.f.was included by Barbhuiya and Salunkhe (2016) in his list orchids of 
Maharashtra citing collection number Dalzell 47 (K-000387793). However, the Dalzell's specimen is identified as Liparis odorata.

Liparis nervosa (Thunb.) Lindl. was included by Almeida (2009) in Flora of Maharashtra and Barbhuiya and Salunkhe (2016) in his list orchids of Maharashtra citing the collection numbers S.M.Almeida 515 (BLAT), Gavade 1475 (BLAT), which was reidentified as Liparis odorata.

Liparis viridiflora (Blume) Lindl. was included by Barbhuiya and Salunkhe (2016) in Orchids of Maharashtra based on collection number Rao $95193(C A L)$. After examining that specimen it was found that the distribution locality is from Karnataka.

Malaxis densiflora (A. Rich.) Kuntze was included by Almeida (2009) in Flora of Maharashtra and Barbhuiya and Salunkhe (2016) in Orchids of Maharashtra citing the collection number B.G.Gavade 441 (BLAT), which was reidentified as Malaxis versicolor.

Peristylus affinis (D.Don) Seidenf. was included by Almeida (2009) in Flora of Maharashtra and Barbhuiya and Salunkhe (2016) in Orchids of Maharashtra citing the collection number S.M.Almeida 4959 (BLAT), which was reidentified as Habenaria marginata.

Peristylus gardneri (Hook.f.) Kraenzl. was included by Almeida (2009) in Flora of Maharashtra and Barbhuiya and Salunkhe (2016) in Orchids of Maharashtra citing the collection number S.M.Almeida 3150 (BLAT), which was reidentified as Peristylus densus.

Peristylus richardianus Wight was included by Almeida (2009) in Flora of Maharashtra and Barbhuiya and Salunkhe (2016) in Orchids of Maharashtra citing the collection number M.R.Almeida 334 (BLAT), which was reidentified as Peristylus densus.

Pholidota pallida Lindl. was included by Almeida (2009) in Flora of Maharashtra and Barbhuiya and Salunkhe (2016) in Orchids of Maharashtra citing the collection number S.M.Almeida 4895 (BLAT), which was reidentified as Pholidota imbricata.

Polystachya concreta (Jacq.) Garay \& H.R.Sweet was included by Barbhuiya and Salunkhe (2016) in Orchids of Maharashtra based on Gammie (1908).
However Gammie (1908) had clearly mentioned that this has not been recorded from Bombay but from the nearby ghats of forest of Belgaum and North Kanara, which are in Karnataka state.

Pteroceras viridiflorum (Thwaites) Holttum was included by Almeida (2009) in Flora of Maharashtra and Barbhuiya and Salunkhe (2016) in Orchids of Maharashtra citing the collection number M.R.Almeida 1621 (BLAT), which was reidentified as Smithsonia viridiflora.

Satyrium nepalense D.Don was included by Almeida (2009) in Flora of Maharashtra and Barbhuiya and Salunkhe (2016) in Orchids of Maharashtra citing the collection number S.M.Almeida 2483 (BLAT), which was reidentified as Peristylus plantagineus.

\section{Doubtful Taxa}

Diplocentrum recurvum Lindl. was included by Lakshminarasimhan et al. (1996) based on L.D.Garade's 257 (BSI) dated 27.05.1902 locality College garden, Poona. There is no other specimen to prove its occurrence in wild in Maharashtra. It requires further intensive survey to confirm its presence.

Peristylus spiralis A.Rich. is based on a collection by Law said to be from the Konkan region. There is no specimen in any herbaria and it was not collected by any other botanist.

\section{Cultivated taxa}

Twenty-two species viz., Coelogyne flaccida, C. nitida, Cymbidium eburneum, Dendrobium albosanguineum, $D$. aphyllum, $D$. cretaceum, $D$. densiflorum, D. farmeri, D. fimbriatum, D. formosum, D. hookerianum, D. lindleyi, D. heterocarpum, D. nobile, D. parishii, D. pulchellum, Epidendrum ellipticum, E. secundum, Phaius mishmensis, P. tankervilleae, Spathoglottis plicata and Vanilla planifolia have been reported by the previous workers from cultivated sources. Six species viz., Arundina graminifolia, Chiloschista lunifera, Goodyera procera, Malaxis acuminata, M. intermedia and M. latifolia were included by Almeida (2009) based on Karthikeyan et al. (1998). However, all these species have no wild record and herbarium specimens to confirm their occurrence in Maharashtra. 
ACKnOwLEDGements. The authors are thankful to Dr. Paramjit Singh, Director, Botanical Survey of India and Dr. P. Lakshminarashiman, Head of Office, Western Regional Centre, Pune for facilities and encouragement. Sincere thanks to Professor M.M.Sardesai for providing herbarium specimens of Gadchiroli district. We are thankful to the authorities of Blatter Herbarium, St. Xavier's College, Mumbai and Shivaji University, Kolhapur Herbarium, Department of Botany, for granting permission to consult their herbarium.

\section{LiTERATURE CITED}

Almeida, M. R. (2009). Flora of Maharashtra-Vol.5a. Mumbai, India: Blatter Herbarium, St. Xavier's College.

Almeida, S. M. (1990). Flora of Sawantwadi Vol. 2. Journal of Economic and Taxonomic Botany Additional Series.

Bachulkar, M. (2010). Addition to the flora of Maharashtra. Journal of Bombay Natural History Society, 107(3), 266.

Bachulkar, M. (2012). Addition to the flora of Maharashtra. Journal of Economic and Taxonomic Botany, 36(2), 381-382.

Bachulkar, M., Sant, N. \& Jagtap, D. (2011). Recollection of a rare orchid Habenaria stenopetala Lindl. from Maharashtra. Life Science Bulletin, 8(2), 277-278.

Bachulkar, M. P. \& Yadav, S. R. (1993). Some new plant records for Maharashtra. Journal of Economic and Taxonomic Botany,

17, 329-331.

Barbhuiya, H. A. \& Salunkhe, C. K. (2016). Orchids of Maharashtra, India: a review. Richardiana, 16, 111-140.

Birdwood, H. M. (1886). A catalogue of the flora of Matheran. Journal of Bombay Natural History Society, 1, $203-214$.

Birdwood, H. M. (1887). A catalogue of the flora of Mahabaleshwar and Matheran. Journal of Bombay Natural History Society, 2, 107-132.

Bhatt, M. R. \& Nagar, P. S. (2014). Aerides ringens (Lindl.) C.E.C.Fisch. -A New Addition to the Orchid Flora of Gujarat, India. Journal of Orchid Society of India, 28, 115-117.

Blatter, E. (1908). The Flora of Bombay Presidency (Statistico-Biological notes). Journal of Bombay Natural History Society, 18, $562-571$

Blatter, E. (1909). The Flora of Panchgani. Journal of Bombay Natural History Society, 19, 314-332.

Blatter, E. (1930). A terrestrial orchid found epiphytic. Journal of Bombay Natural History Society, 34, 599.

Blatter, E. \& McCann, C. (1931a). Revision of the flora of the Bombay Presidency -Orchidaceae-Part 16. Journal of Bombay Natural History Society, 35, 254-275.

Blatter, E. \& McCann, C. (1931b). Revision of the flora of the Bombay Presidency -Orchidaceae-Part 17. Journal of Bombay Natural History Society, 35, 484-495.

Blatter, E. \& McCann, C. (1931c). Revision of the flora of the Bombay Presidency -Orchidaceae-Part 18. Journal of Bombay Natural History Society, 35, 722-735.

Blatter, E. \& McCann, C. (1932). Revision of the flora of the Bombay Presidency -Orchidaceae-Part 19. Journal of Bombay Natural History Society, 36, 13-28.

Cerejo-Shivkar, S. \& Shinde, R. D. (2015). A brief account of Orchidaceae in Sanjay Gandhi National Park, Mumbai, India. Journal of Threatened Taxa, 7(6), 7287-7295.

Champion, H. G. \& Seth, S. K. (1968). A revised survey of the forest types of India. Delhi, India: Manager of Publications. Cooke, T. (1907). Flora of the Presidency of Bombay. Vol. II. London, England: Taylor and Francis.

Dalzell, A. N. \& Gibson, A. (1861). The Bombay Flora. Bombay, India: Education Society's Press.

Gaikwad, S. P., Gore, R. D. \& Garad, K.U. (2012). Additions to the flora of Marathwada region of Maharashtra, India. Journal of Threatened Taxa, 4(4), 2515-2523.

Datar, M. N. \& Ghate,V. S. (2012). Extended distribution of Smithsonia straminea C.J.Saldanha, an endemic orchid in Maharashtra, India. Journal of Threatened Taxa, 4(2), 2406-2408.

Gammie, G. A. (1904a). The Orchids of Bombay Presidency. Journal of Bombay Natural History Society, 16, 429-433.

Gammie, G. A. (1904b). The Orchids of Bombay Presidency. Journal of Bombay Natural History Society, 16, 562-569.

Gammie, G. A. (1906). The Orchids of Bombay Presidency. Journal of Bombay Natural History Society, 17, 31-37.

Gammie, G. A. (1907a). The Orchids of Bombay Presidency. Journal of Bombay Natural History Society, 17, 940-942.

Gammie, G. A. (1907b). The Orchids of Bombay Presidency. Journal of Bombay Natural History Society, 18, 88-91. 
Gammie, G. A. (1908a). The Orchids of Bombay Presidency. Journal of Bombay Natural History Society, 18, 586-590. Gammie, G. A. (1908b). The Orchids of Bombay Presidency. Journal of Bombay Natural History Society, 18, 833-834. Gammie, G. A. (1909a). The Orchids of Bombay Presidency. Journal of Bombay Natural History Society, 19, $139-141$. Gammie, G. A. (1909b). The Orchids of Bombay Presidency. Journal of Bombay Natural History Society, 19, $624-626$. Gammie, G. A. (1910). The Orchids of Bombay Presidency. Journal of Bombay Natural History Society, 20, 126-129. Gammie, G. A. (1911a). The Orchids of Bombay Presidency. Journal of Bombay Natural History Society, 20, 597-602. Gammie, G. A. (1911b). The Orchids of Bombay Presidency. Journal of Bombay Natural History Society, 21, $171-174$. Gammie, G. A. (1912). The Orchids of Bombay Presidency. Journal of Bombay Natural History Society, 21, 1129-1130.

Govaerts, R., Pernet, P., Kratochvil, K., Gerlach, G., Carr, G., Alrich, P., Pridgeon, A. M., Pfahl, J., Campacci, M. A., Baptista, D. H., Tigges, H., Shaw, J., Cribb, P., George, A., Kreuz, K. \& Wood, J. (2016). World Checklist of Orchidaceae. Kew: Royal Botanic Gardens. Retrieved from http://apps.kew.org/wcsp/ (accessed 22 May 2016).

Graham, J. (1839). A catalogue of the plants growing in Bombay and its vicinity. Bombay, India: Government Press.

IPNI (2016). The International Plant Names Index. Available from http://www.ipni.org.

Jain, S.K. \& Rao, R.R. (1960). A handbook of field and Herbarium methods. New Delhi, India: Today and tomorrow's publishers. Jalal, J. S. \& Jayanthi, J. (2013). Does Habenaria panchganiensis Santapau \& Kapadia exist? Taiwania, 58(4), 316-320.

Jalal, J. S., Jayanthi, J. \& Kumar, P. (2013). A note on the morphological variations in Rhynchostylis retusa (Orchidaceae) in Maharashtra, India. McAllen International Orchid Society Journal, 15(11), 16-20.

Jalal, J. S., Jayanthi, J. \& Kumar, P. (2014). Eulophia spectablis: A high value medicinal orchid under immense threat due to overexploitation for medicinal uses in Western Ghats, Maharashtra, India. McAllen International Orchid Society Journal, 15(10), 9-15.

Kamble, S. Y. \& Pradhan, S. G. (1988). Flora of Akola District, Maharashtra. Howrah, India: Botanical Survey of India, Department of Environment.

Kothari, M. J. \& Moorthy, S. (1993). Flora of Raigad District, Maharashtra State. Calcutta, India: Botanical Survey of India, Department of Environment.

Kulkarni, B. G. (1988). Flora of Sindhudurg. Howrah, India: Botanical Survey of India, Department of Environment.

Lakshminarasimhan, P. \& Sharma, B.D. (1991). Flora of Nasik District. Calcutta, India: Botanical Survey of India, Department of Environment.

Lakshminarasimhan, P., Sharma, B. D., Karthikeyan, S. \& Singh, N. P. (eds.). (1996). Flora of Maharashtra State: Monocotyledones. Flora of India Series 2. Calcutta, India: Botanical Survey of India, Department of Environment.

Mudaliar, S. K. (1991). Peristylus constrictus (Lindl.) Lindl., a new record for Maharashtra. Journal of Economic and Taxonomic Botany, 15(2), 469.

Mulgaonkar, S. M. \& Dabhade, G. T. (2005). Some observations on the pollinia of the three species of corticolous orchid of the genus Aerides Lour under SEM. Indian Journal of Applied and Pure Biology, 20(1), 43-46.

Nairne, A. K. (1894). The Flowering Plants of Western India. London, England: W. H. Allen And Company Limited.

Nayar, T. S. \& Kochhar, R. K. (1984). A new record of Habenaria Willd. For Maharashtra state. Journal of Economic and Taxonomic Botany, 5, 920.

Pande, S., Sant, N., Vishwarao, V. \& Datar, M. N. (2010). Wild orchids of Northern Western Ghats (including Maharashtra, Goa and Belgaum). Pune, India: Ela Foundation.

Pradhan, S. G., Sharma, B. D. \& Singh, N. P. (2005). Flora of Sanjay Gandhi National Park, Borivali-Mumbai (Bombay). Calcutta, India: Botanical Survey of India, Department of Environment.

Prabhu, S. (2013). Neglected Habitats: Coastal Plateau. Hornbill, 1, 38-44.

Punekar, S. A. (2002a). Rediscovery of Zeuxine longilabris (Lind1.) Benth. (Orchidaceae)- A little known species. Journal of Economic and Taxonomic Botany, 26, 108-110.

Punekar, S. A. (2002b). Report of rare terrestrial orchid species Cheirostylis parvifolia Lindl. from Dajipur Wildlife Sanctuary, Kolhapur district of Maharashtra state, India, Journal of Economic and Taxonomic Botany, 26, 105-107.

Sanghamitra, M. R., Raut, S. V. \& Mulgaonkar, M. S. (2012). Survey of some medicinally important epiphytic orchids from Yeoor in Thane. Phytotaxonomy, 12, 155-159. 
Santapau, H. \& Kapadia, Z. (1957). Habenaria panchganiensis - new name for a Bombay orchid. Journal of Bombay Natural History Society, 54, 478.

Santapau, H. \& Kapadia, Z. (1966). The Orchids of Bombay. Delhi, India: Manager of Publications.

Sardesai, M. M. \& Yadav, S. R. (2004). Genus Habenaria Willd. (Orchidaceae) in Maharashtra, pp. 144-172. In: T. Pullaiah (ed.). Biodiversity in India. Vol. III. New Delhi, India: Regency Publications.

Sardesai, M. M., Bachulkar, M. P. \& Yadav, S. R. (2002). Additions to orchid flora of Maharashtra. Journal of Bombay Natural History Society, 99, 564-566.

Singh, N. P. \& Dawre, M. S. (1983). On the Occurrence of Acampe rigida (Buch.-Ham. Ex J.E.Sm.) Hunt in Peninsular India. Journal of Ecomomic Taxonomy and Botany, 4(3), 1021-1022.

Shah, G. L. \& Badrinath, V. (1985). A contribution to the angiospermic flora of Dahanu Forest Division in Maharashtra State. Journal of Economic and Taxonomic Botany, 6(1), 117-141.

The Plant List (2013). Version 1.1. Published on the Internet; http://www.theplantlist.org/ (accessed 1st January).

Tropicos (2016). Missouri Botanical Garden. Available from www.tropicos.org.

Ugemuge N.R. (1986). Flora of Nagpur District. Shree Publication, Nagpur.

Watve, A. (2013). Status review of Rocky Plateaus in the northern Western Ghats and Konkan region of Maharashtra, India with recommendations for conservation and management. Journal of Threatened Taxa, 5(5), 3935-3962. http://dx.doi.org/10.11609/ JoTT.o3372.3935-62

Woodrow, G.M. (1898-1900). The flora of Western India. Journal of Bombay Natural History Society, 12, 515-526. 